Research Paper

\title{
Cold Inducible RNA Binding Protein Is Involved in Chronic Hypoxia Induced Neuron Apoptosis by Down-Regulating HIF-1 $\alpha$ Expression and Regulated By microRNA-23a
}

\author{
Xiaoming Chen*, Xinqin Liu*, Bin Li*, Qian Zhang ${ }^{*}$, Jiye Wang, Wenbin Zhang ${ }^{\bowtie}$, Wenjing Luo ${ }^{\bowtie}$, Jingyuan Chen ${ }^{\bowtie}$ \\ Department of Occupational and Environmental Health, the Ministry of Education Key Lab of Hazard Assessment and Control in Special Operational \\ Environment, School of Public Health, Fourth Military Medical University, Xi'an, China \\ * These authors contributed equally to this work. \\ $\bowtie$ Corresponding authors: Wenjing Luo, e-mail: luowenj@fmmu.edu.cn; Wenbin Zhang, e-mail: zwb@fmmu.edu.cn; and Jingyuan Chen, e-mail: \\ jy_chen@fmmu.edu.cn. \\ (1) Ivyspring International Publisher. This is an open access article distributed under the terms of the Creative Commons Attribution (CC BY-NC) license \\ (https://creativecommons.org/licenses/by-nc/4.0/). See http://ivyspring.com/terms for full terms and conditions.
}

Received: 2016.10.03; Accepted: 2017.02.27; Published: 2017.04.10

\begin{abstract}
Background: Neuron apoptosis mediated by hypoxia inducible factor $1 \alpha(\mathrm{HIF}-1 \alpha)$ in hippocampus is one of the most important factors accounting for the chronic hypobaric hypoxia induced cognitive impairment. As a neuroprotective molecule that is up-regulated in response to various environmental stress, CIRBP was reported to crosstalk with HIF-1a under cellular stress. However, its function under chronic hypobaric hypoxia remains unknown.

Objective: In this study, we tried to identify the role of CIRBP in HIF-l $\alpha$ mediated neuron apoptosis under chronic hypobaric hypoxia and find a possible method to maintain its potential neuroprotective in long-term high altitude environmental exposure.

Methods: We established a chronic hypobaric hypoxia rat model as well as a tissue culture model where SH-SY5Y cells were exposed to $1 \%$ hypoxia. Based on these models, we measured the expressions of HIF- $1 \alpha$ and CIRBP under hypoxia exposure and examined the apoptosis of neurons by TUNEL immunofluorescence staining and western blot analysis of apoptosis related proteins. In addition, by establishing HIF-I $\alpha$ shRNA and PEGFP-CIRBP plasmid transfected cells, we confirmed the role of HIF-l $\alpha$ in chronic hypoxia induced neuron apoptosis and identified the influence of CIRBP over-expression upon HIF-l $\alpha$ and neuron apoptosis in the process of exposure. Furthermore, we measured the expression of the reported hypoxia related miRNAs in both models and the influence of miRNAs' over-expression/knock-down upon CIRBP in the process of HIF-l $\alpha$ mediated neuron apoptosis.

Results: HIF-1a expression as well as neuron apoptosis was significantly elevated by chronic hypobaric hypoxia both in vivo and in vitro. CIRBP was induced in the early stage of exposure (3d/7d); however as the exposure was prolonged (21d), CIRBP level of the hypoxia group became significantly lower than that of control. In addition, HIF-la knockdown significantly decreased neuron apoptosis under hypoxia, suggesting HIF-la may be pro-apoptotic in the process of exposure. CIRBP over-expression significantly suppressed HIF-1 $\alpha$ up-regulation in hypoxia and inhibited HIF-l a mediated neuron apoptosis. Interestingly, miR-23a was also induced by hypoxia exposure and showed the same changing tendency with CIRBP (increasing in $3 \mathrm{~d} / 7 \mathrm{~d}$, decreasing in $21 \mathrm{~d}$ ). In addition, over-expressing miR-23a up-regulated CIRBP, down-regulated HIF-1 $\alpha$ and attenuated neuron apoptosis.

Conclusion: Cold inducible RNA binding protein is involved in chronic hypoxia induced neuron apoptosis by down-regulating HIF-1 $\alpha$ expression, and MiR-23a may be an important tool to maintain CIRBP level and function.
\end{abstract}

Key words: chronic hypobaric hypoxia, hypoxia inducible factor, cold inducible RNA binding protein, microRNA. 


\section{Introduction}

Chronic hypobaric hypoxia encountered at high altitude is known to induce cognitive function impairment, which persisted even after returning to sea level [1-5]. Cognitive functions, like learning and memory, are an important attribute of the hippocampus. Previous studies have demonstrated that severe and chronic (> $5500 \mathrm{~m}$, for 3-4d) hypoxia caused neuronal death in the CA3, CA4 and dentate gyrus of the hippocampus [6,7], indicating that neuron apoptosis in this brain region would be one of the prominent factors accounting for chronic hypobaric hypoxia induced cognitive impairment.

HIF-1a is the most important transcription factor in cellular hypoxia response, who is closely linked to hypoxia induced neuron apoptosis $[8,9]$. Under hypoxia stress, HIF-1a could initiate neuron apoptosis by increasing the binding proteins of anti-apoptotic protein $\mathrm{Bcl}-2$ thereby inhibiting its anti-apoptotic effect [10-12]. Moreover, HIF-1a was also relevant to neuronal apoptosis after brain injury by regulating p53 and BNIP3 in the apoptotic neurons [13-17]. Herein, in order to attenuate the hypoxia induced neuron apoptosis in cognition related brain region, it's medically important to portrait the detailed regulating mechanism of HIF-1a under hypoxia stress and find the protective factor.

Cold-inducible RNA-binding protein (CIRBP) was initially screened as a gene transcript that is induced by DNA damage and plays a key role in controlling cellular responses to various environmental stress like hypothermia and ultraviolet light [18-20]. Several studies have reported that under environmental stress, CIRBP migrates from nucleus to cytoplasm, regulating its target mRNAs at the post-transcriptional level and exert neuroprotective effect [21-24]. For example, CIRBP may inhibit neuron apoptosis through the suppression of the mitochondria apoptosis pathway during mild hypothermia [25, 26]. Moreover, the induction of CIRBP protein in rat cortical neurons under hypothermia inhibits $\mathrm{H}_{2} \mathrm{O}_{2}$-induced neuronal apoptosis, which forms one of the cerebral protective pathways under hypothermia [27]. It has been reported that, CIRBP was up-regulated in response to acute mild $\left(8 \% \mathrm{O}^{2}\right)$ or severe $\left(1 \% \mathrm{O}^{2}\right)$ hypoxia [28]. However, the expression characteristics of CIRBP in the brain under chronic hypobaric hypoxia are still unknown [29], whether CIRBP could function as a neuroprotective factor in chronic hypobaric hypoxia remains unconfirmed.

The association between CIRBP and HIF-1a has been discussed, and there is several conclusions supporting their crosstalk under stress. Elizabeth T. Chang found that CIRBP could bind to HIF-1a and several protein translation factor mRNAs on polysomes and increase de novo protein translation under cellular stress [30]. Considered the important role of CIRBP in stress induced neuron apoptosis, we hypothesize that as a neuroprotective factor, CIRBP may take part in the regulation of HIF-1a mediated neuron apoptosis and exert neuroprotective function under chronic hypobaric hypoxia.

Micro(mi)RNAs are small noncoding RNAs that play crucial roles in regulating various biological processes, including cell differentiation, proliferation, and apoptosis [31]. A family of hypoxia-sensitive miRNAs, named hypoxamirs, is induced when cells experience low-oxygen conditions, and these miRNAs specifically participate in controlling a network of multiple processes such as oncogenesis, angiogenesis and cell apoptosis [32-34]. Previous study showed that miR-23 could mediate hypoxia-induced apoptosis of myocardial cells [35]. Besides, miR-1 could mediate acute hypoxia-induced apoptotic insults to neuro-2a cells via an intrinsic Bax-mitochondrion-caspase protease pathway [36], while miR-23a can regulate the sensitivity of neurons to apoptosis by increasing endogenous inhibitory factors [37]. However, the roles of miR-1 and miR-23 in chronic hypobaric hypoxia-induced neuron apoptosis and their possible relation with CIRBP are little known.

In order to confirm our hypothesis, we measured the dynamic change of CIRBP/HIF-1a expression and neuron apoptosis in the hippocampus of chronic hypobaric hypoxia exposed rats and $1 \%$ hypoxia exposed SH-SY5Y cells. To investigate the potential association between the change of CIRBP and hypoxia induced neuron apoptosis, we examined the effect of CIRBP over-expression on HIF-1a expression and the ratio of neuron apoptosis. Moreover, in order to seek the methods to maintain the level and potential neuroprotective function of CIRBP, we measured the hypoxia related miRNAs in hypoxia models and explored their influence upon CIRBP/HIF-1a expression and neuron apoptosis. Through this study, we try to identify the role of CIRBP in HIF-1a mediated neuron apoptosis under chronic hypobaric hypoxia and find a reliable method to maintain its potential neuroprotective in long-term high altitude environmental exposure. To our knowledge, this may be the first study focusing on the potential neuroprotective function of CIRBP in the field of environmental health. 


\section{Materials and Methods}

\section{In vivo hypobaric hypoxia animal models}

Adult male Sprague-Dawley rats ( $\mathrm{n}=40$ and 3 months old) with an average body weight of $225 \pm 25 \mathrm{~g}$ were used for this study. Before hypobaric hypoxia exposure, all rats were maintained in the institute animal house, exposed to $12 / 12 \mathrm{~h}$ light/dark cycles, provided with pellet diet and water ad libitum. The ethics committee of the institute approved all experimental protocols for this study and adequate measures were taken to minimize pain or discomfort to the rats. The rats were randomly divided into three control (normoxic) group and three hypoxia group (6 animals in each group, 3 for TUNEL staining and 3 for western blot analysis): 1) The three control (normoxic) groups was kept at normal atmospheric pressure (i.e. sea level) for $3 d, 7 d$ and $21 d$, respectively, 2) The hypoxia groups were exposed to an altitude of $6100 \mathrm{~m}$ (barometric pressure $=349 \mathrm{~mm} \mathrm{Hg}$ and partial $\mathrm{O}_{2}$ pressure $\sim 8-9 \%$ ) continuously for $3 \mathrm{~d}, 7 \mathrm{~d}$ and $21 \mathrm{~d}$. Simulated high altitude was created in a specially designed decompression chamber, which enables reduction of barometric pressure (e.g. $349 \mathrm{~mm} \mathrm{Hg}$ ). Temperature and humidity were maintained at 22-26 ${ }^{\circ} \mathrm{C}$ and $55-60 \%$ respectively. The decompression chamber was continuously supplied with fresh air to replenish $\mathrm{O}_{2}$ consumed by the rats and to flush out exhaling carbon dioxide. The desired altitude was attained at a rate of $300 \mathrm{~m} / \mathrm{min}$ over a period of 20 min. Pressure of the decompression chamber was brought to sea level daily at 10 a.m. for $30 \mathrm{~min}$ at a rate of $300 \mathrm{~m} / \mathrm{min}$ to replenish food and water. During exposure, rats of all groups were all kept with 12/12 h light/dark cycles and provided with pellet diet and water ad libitum.

\section{Immunofluorescence}

The animals were deeply anesthetized and then transcardially perfused with $100 \mathrm{ml}$ saline solution, followed by $400 \mathrm{ml} 4 \%$ paraformaldehyde (PFA) solution. The brains were removed and post-fixed overnight in PFA. The fixed brains were dehydrated in alcohol and embedded in paraffin and 8- $\mu \mathrm{m}$-thick slices were cut from the paraffin-embedded tissues, washed three times in $0.01 \mathrm{M}$ PBS, then permeabilized in $0.5 \%$ Triton X-100 in PBS. The sections were then immersed in $0.5 \% \mathrm{H}_{2} \mathrm{O}_{2}$ in methanol for $10 \mathrm{~min}$ to block endogenous peroxidases and non-specific binding sites were blocked with $5 \%$ non-fat milk in PBS for $1 \mathrm{~h}$ at room temperature. Afterward, the sections were incubated with primary antibodies overnight at $4 \mathrm{C}$. Finally, the immunoreaction was detected using FITC or PE-conjugated secondary antibodies. The images were visualized with a fluorescence microscope. Mouse polyclonal antibodies NeuN (Cat. number: 128886, 1:200), rabbit polyclonal antibodies caspase 3 (Cat. number: 52293, 1:200), cleaved caspase 3 (Cat. number: ab2302,1:200) were obtained from Abcam (Cambridge, UK).

\section{In situ TUNEL apoptosis detection}

To assess DNA fragmentation, consecutive sections were processed by TUNEL using an in situ cell death detection kit (Roche). The TUNEL technique was applied as described by Whiteside, G and colleagues [38,39]. Briefly, the fixed brains were dehydrated in alcohol and embedded in paraffin. Next, 10- $\mu$-thick slices were cut from the paraffin-embedded tissues, washed three times in 0.01 $\mathrm{M}$ PBS, then permeabilized in proteinase $\mathrm{K}$ for $10 \mathrm{~min}$. After another three washes, the sections were incubated in TdT buffer at $37^{\circ} \mathrm{C}$ for $1 \mathrm{~h}$ and then with antibody at $37^{\circ} \mathrm{C}$ for $1 \mathrm{~h}$. Afterward, the sections were incubated with primary antibody NeuN overnight at 4 C. Finally, the immunoreaction was detected using PE-conjugated secondary antibodies. The sections were stained by Hoechst 33342 counterstaining and mounted on a using a fluorescence microscope. Five random slides were selected from each group, and from each slide, five randomly selected visual fields in the hippocampus CA3 region were observed at a magnification of $\times 20$. The number of TUNEL/NeuN-positive cells was counted.

\section{Western blot analysis}

After stipulated period of exposure, three rats in each group were decapitated and western blot was used to determine the levels of HIF-1a, CIRBP, cleaved caspase-3/caspase-3, Bax/Bcl-2 in hippocampus. The animals were quickly sacrificed by cervical dislocation. The brain was removed and the hippocampus were dissected out in the ice-cold $0.9 \%$ $\mathrm{NaCl}$ solution. All dissected tissues were kept in liquid nitrogen. Tissue samples were homogenized in ice-cold lysis buffer containing $50 \mathrm{mM}$ Tris- $\mathrm{HCl}(\mathrm{pH}$ 7.4), $1 \%$ NP-40, $0.25 \%$ na-deoxycholate, $150 \mathrm{mM} \mathrm{NaCl}$, $1 \mathrm{mM}$ PMSF, $1 \mathrm{mM}$ EDTA, $1 \mathrm{mg} / \mathrm{ml}$ pepstatin, $1 \mathrm{mM}$ $\mathrm{Na} 3 \mathrm{VO} 4$ and $1 \mathrm{mM} \mathrm{NaF}$. Crude homogenates were incubated on ice for $30 \mathrm{~min}$ and centrifuged at 15,000 $\mathrm{g}$ for $30 \mathrm{~min}$ at $4{ }^{\circ} \mathrm{C}$. The supernatant was collected for protein assay and stored at $-70{ }^{\circ} \mathrm{C}$. Protein concentration was determined by the Bicinchoninic Acid Kit protein assay (Pierce Chemical Co.). Equal amounts of lysates were separated by $10 \%$ SDS-polyacrylamide gel electrophoresis and electro-transferred onto nitrocellulose membranes. Membranes were then incubated in blocking solution (5\% non-fat milk in $20 \mathrm{mM}$ TRIS-HCl, $150 \mathrm{mM} \mathrm{NaCl}$, $0.1 \%$ Tween-20) (TBS-T), followed by incubation with 
the indicated antibodies at $4^{\circ} \mathrm{C}$ overnight. The membranes were then washed in TBS-T and incubated with HRP-conjugated secondary antibodies for $1 \mathrm{~h}$ at room temperature. Enhanced chemiluminescence (ECL) Western Blotting Substrate (Pierce) was used to detect the immunoreactive signals with an ECL-based FluorChem FC2 image system (Alpha Innotech). Rabbit anti-CIRBP (Cat. number: 10209-2-AP) was purchased from ProteinTech. Rabbit anti-HIF-1a (Cat. number: 14179), rabbit anti-Bax (Cat. number: 14796), mouse anti-Bcl-2 (Cat. number: 15071), anti-caspase-3 (Cat. number: 9662) and anti-cleaved caspase-3 (Cat. number: 9664) were purchased from Cell Signaling Technology (Beverly, MA, USA). Image J software was used to analyze the gray values of the bands in each group. Each protein band was normalized to $\beta$-actin values and expressed as the intensity ratio. All western blot analyses were performed in triplicates.

\section{In vitro chronic $1 \%$ hypoxia cell model}

\section{Cells culture and hypoxia exposure}

Human neuronal-like SH-SY5Y neuroblastoma cells line was cultured in Roswell Park Memorial Institute (RPMI) 1640 Medium containing $2 \mathrm{mM}$ L-glutamine (Invitrogen, USA), supplemented with $10 \%$ heat-inactivated FBS and $100 \mathrm{U} / \mathrm{mL}$ penicillin/streptomycin. The cultures were maintained in a standard humidified incubator in 5\% $\mathrm{CO} 2$ at $37^{\circ} \mathrm{C}$, with fresh medium replaced every $2 \mathrm{~d}$, and split 1:4 when the cells reached $90 \%$ confluence. Hypoxia environment was made by placing cells in a humidified microaerophilic incubation system (DWS HypOxystation) with a calibrated gas containing 1\% $\mathrm{O} 2$, or $3 \% \mathrm{O} 2$ at $37^{\circ} \mathrm{C}(\mathrm{CO} 2$ was adjusted at $5 \%$ in both conditions). The cells were left in the incubator at 37 ${ }^{\circ} \mathrm{C}$ for different durations. The control cultures were incubated in normoxic conditions all the time for the same durations.

\section{TUNEL assay}

To evaluate cell apoptosis, TdT-dUTP nick-end labeling (TUNEL) assays were performed using a one step in situ cell death detection kit (Roche, Germany) according to the manufacturer's instructions. Briefly, after the induction of apoptosis, cells were fixed with $4 \%$ paraformaldehyde in PBS ( $\mathrm{pH} 7.4$ ) for $1 \mathrm{~h}$ at room temperature, washed in PBS, and then incubated with $0.1 \%$ Triton X-100 for 2 min on ice. Later, the cells were incubated in TUNEL reaction mixture in a humidified atmosphere for $1 \mathrm{~h}$ at $37^{\circ} \mathrm{C}$ in the dark. Hoechst 33342 (1:5000, Invitrogen, USA) was used to label nuclei. TUNEL-positive cells were imaged under a fluorescence microscope. Cells showing red fluorescence were considered apoptotic cells.

\section{Flow cytometry analysis for apoptosis}

In parallel, flow cytometry was used to further analyze cell apoptosis. After hypoxia exposure, SH-SY5Y cell samples were trypsinized and then centrifuged at $2000 \mathrm{rpm}$ for $5 \mathrm{~min}$. The cells were resuspended with $500 \mathrm{ml}$ binding buffer at a concentration of 106 cells $/ \mathrm{ml}$, after washing two times with PBS at $2000 \mathrm{rpm}$ for $5 \mathrm{~min}$. Then, $5 \mathrm{ml}$ FITC-conjugated Annexin-V and $5 \mathrm{ml}$ PI were added to the cells and incubated at room temperature for 15 min in the dark. The samples were analyzed within 1 $\mathrm{h}$ post-staining.

\section{Lentivirus stable transduction}

In order to get HIF-1a knockdown SH-SY5Y cell line, we generated stable SH-SY5Y cell lines by lentiviral transduction and antibiotic selection that express control or HIF-1a miR30 shRNA. Lentiviruses which were generated by co-transfection of shuttle vectors with packaging plasmids $\mathrm{pMD} 2 \mathrm{G}$, were gifted from Dr. Rui Chen and described before [40]. In brief, the day before transduction, SH-SY5Y cells were trypsinized and $2 \times 10^{5}$ cells per well plated in $1 \mathrm{~mL}$ complete culture medium in a 6-well plate for overnight incubation at $37^{\circ} \mathrm{C}$. On the day of transduction, media was removed and replaced with $1 \mathrm{ml}$ of complete medium with $10 \mu \mathrm{g} / \mathrm{ml}$ polybrene (Cat: 107689, Sigma). Lentiviral particles were thawed to room temperature, mixed gently, and added to the HT1080 cells $(\mathrm{MOI}=30)$. After gently swirling to mix, cells were incubated overnight. After $12 \mathrm{~h}$, culture medium was replaced with $2 \mathrm{ml}$ of complete medium containing 800 mg/1 G418(Cat: A1720, Sigma), which was replaced every $2 \mathrm{~d}$ until one week after all control cells had died. Positive cells were maintained in 500 $\mathrm{mg} / \mathrm{l} \mathrm{G} 418$ for 2 weeks, and then were frozen down until use. For experiments, cells were thawed and allowed to grow for three passages before use.

\section{Plasmid construction and transfection}

Human CIRBP cDNA clone (NC_000019) in pEGFP-N2 vector was constructed by Dr. HongLei Che and described by Zhang $Q$ before [41]. The control transfection was performed by pEGFP-N2 vector without CIRBP. Overexpression of CIRBP in the cells was verified by western blot with anti-CIRBP antibody as descripted below. Transfection of SH-SY5Y cells with CIRBP cDNA was performed by with Lipofectamine 2000 transfection reagent (Invitrogen, USA), according to the manufacturer's procedure. In brief, cells were plated in 6-well plates (Nunc) at a cell density of $3 \times 10^{5}$ cells per well and were allowed to grow overnight to achieve $80 \%$ confluency. Transfection complexes, consisting of 2.5 ug pEGFP-N2 vector plasmid DNA or 
pEGFP-N2-CIRBP plasmid DNA and $6 \mathrm{uL}$ Lipofectamine reagent, were added to the wells in Opti-MEM® Medium (Invitrogen, USA). Cells were analyzed $48 \mathrm{~h}$ after lipofection for transfection efficiency and viability.

\section{Oligonucleotides transfection}

Transfections of SH-SY5Y cells with oligonucleotides (miR-23a mimics, miR-23a inhibitors, miR-1 mimics and miR-1 inhibitors) (Shanghai Jima Gene Biotech Co., Shanghai) were used at a final concentration of $20 \mathrm{nM}$ in antibiotic-free Opti-MEM medium (Invitrogen, USA). Transfection was performed with Lipofectamine 2000 reagent (Invitrogen, USA), following the manufacturer's protocol. Briefly, $3 \times 10^{5}$ cells were seeded in 6-well plates one day before transfection to ensure suitable cell confluency on the day of transfection. The complex was added dropwise to a 6-well cell culture plate with replaced medium, mixed by agitating, and placed in a $\mathrm{CO}_{2}$ incubator at $37^{\circ} \mathrm{C}$. Transfection efficiency was detected after 4-6 $\mathrm{h}$, and the entire operation was protected from light. The sequences of miRNA inhibitors and mimics were as follows:

hsa-miR-1 inhibitor:

5'-AUACAUACUUCUUUACAUUCCA-3';

hsa-miR-1 mimics:

5'-ACAUACUUCUUUACAUUCCAUU-3';

hsa-miR-23a inhibitor:

5'-GGAAAUCCCUGGCAAUCUGAU-3';

hsa-miR-23a mimics:

5'-AAAUCCCUGGCAAUGUGAUUU-3';

negative control for miRNA inhibitors and mimics: 5'-CAGUACUUUUGUGUAGUACAA -3'.

\section{Western blot analysis}

Cells were washed twice with ice-cold PBS and lysed with buffer containing TRIS- $\mathrm{HCl}(50 \mathrm{mM}, \mathrm{pH}=$ 7.4), NP-40 (1\%), Na-deoxycholate (0.25\%), NaCl (150 $\mathrm{mM})$, EDTA (1 mM), PMSF (1 mM), Na3VO4 (1 mM), $\mathrm{NaF}(1 \mathrm{mM})$ for total extract. Protein concentration was determined by the Bicinchoninic Acid Kit protein assay (Pierce Chemical Co.). Equal amounts of cell lysates were separated by $10 \%$ SDS-polyacrylamide gel electrophoresis and electro-transferred onto nitrocellulose membranes. Membranes were then incubated in blocking solution (5\% non-fat milk in 20 $\mathrm{mM}$ TRIS- $\mathrm{HCl}, 150 \mathrm{mM} \mathrm{NaCl}, 0.1 \%$ Tween-20) (TBS-T), followed by incubation with the indicated antibodies at $4^{\circ} \mathrm{C}$ overnight. The membranes were then washed in TBS-T and incubated with HRP-conjugated secondary antibodies for $1 \mathrm{~h}$ at room temperature. Enhanced chemiluminescence (ECL) Western Blotting Substrate (Pierce) was used to detect the immunoreactive signals with an ECL-based
FluorChem FC2 image system (Alpha Innotech). Image J software was used to analyze the gray values of the bands in each group. Rabbit anti-CIRBP (Cat. number: 10209-2-AP) was purchased from ProteinTech. Rabbit anti-HIF-1a (Cat. number: 14179), rabbit anti-Bax (Cat. number: 14796), mouse anti-Bcl-2 (Cat. number: 15071), anti-caspase-3 (Cat. number: 9662) and anti-cleaved caspase-3 (Cat. number: 9664) were purchased from Cell Signaling Technology (Beverly, MA, USA). Image J software was used to analyze the gray values of the bands in each group. Each protein band was normalized to $\beta$-actin values and expressed as the intensity ratio. All western blot analyses were performed in triplicates.

\section{Real-time PCR for miRNA expression}

For in vivo chronic hypobaric hypoxia animal model, total RNA was extracted from cells using TRIzol reagent (Invitrogen), according to the manufacturer's instructions. For in vitro miRNA analysis, mature miRNA was extracted from cell lines with or without hypoxia exposure (as described above). RNA concentrations and integrity were determined using the NanoDrop 2000 Spectrophotometer (Thermo Fisher Scientific, MA). MiRNAs were reverse-transcribed with the One Step PrimeScript miRNA cDNA Synthesis Kit (TaKaRa Code: D350A), according to the manufacturer operating instructions, and the reaction system was vibrated and centrifuged briefly, then incubated at $37^{\circ} \mathrm{C}$ for $15 \mathrm{~min}$ and $85^{\circ} \mathrm{C}$ for $5 \mathrm{~s}$. After reaction, the system was centrifuged briefly again and placed on ice to cool. Real-time PCR was performed using SYBR Green assays in ABI7500 system. U6 snRNA was not responsive to hypoxia and stable among all cell-lines thus was used as the endogenous control. The reaction conditions for SYBR Green quantitative PCR were: pre-denaturation at $95^{\circ} \mathrm{C}$ for $10 \mathrm{~min}$; followed by 40 cycles of denaturation at $95^{\circ} \mathrm{C}$ for $15 \mathrm{~s}$, annealing at $60^{\circ} \mathrm{C}$ for $20 \mathrm{~s}$, and extension at $72^{\circ} \mathrm{C}$ for $20 \mathrm{~s}$. The melting curve was investigated immediately after the reaction was completed using the following regimen: $95^{\circ} \mathrm{C}$ for $15 \mathrm{~s}, 60^{\circ} \mathrm{C}$ for $1 \mathrm{~min}, 95^{\circ} \mathrm{C}$ for $15 \mathrm{~s}$, and $60^{\circ} \mathrm{C}$ for $15 \mathrm{~s}$. The primer sequences were 5'-CGTGGAATGTAAAGAAGTGTGT-3' for miR-1, and $5^{\prime}$-CATCACATTGCCAGGGATTTC-3' for miR-23a. The cycle threshold $(\mathrm{Ct})$ values for each well were determined and the $\Delta \Delta \mathrm{Ct}$ values were calculated using the following equation: $\Delta \Delta \mathrm{Ct}=\left(\mathrm{Ct}_{\text {sample }}\right.$ $\mathrm{Ct}_{\text {reference }}$ gene $)$ - $\left(\mathrm{Ct}_{\text {negative }}\right.$ control - $\mathrm{Ct}_{\text {reference }}$ gene). After standardization, the $2(\Delta \Delta \mathrm{Ct})$ values were calculated, which represented the value of the initial copy number of the sample. 


\section{Statistical analysis}

Continuous variables were expressed as mean \pm standard error (SEM) and analyzed using the student's $t$-test. All statistical analysis was done using GraphPad Prism 5.0 (GraphPad Software, Inc. San Diego, CA). A $p$ value of less than 0.05 was considered statistically significant.

\section{Results}

\section{Chronic hypobaric hypoxia exposure leads to elevated ratio of neuron apoptosis and significant change of CIRBP expression in rat hippocampus}

Previous research has reported that hypobaric hypoxia exposure will lead to persistent low oxygen partial pressure in deep cerebral cortical region [42-44]. To explore the effect of chronic hypoxia exposure on hippocampal neurons, which were reported to be vulnerable to hypoxia, we kept the adult rats in animal decompression chamber at the pressure of $349 \mathrm{mmHg}$ for $3 \mathrm{~d}, 7 \mathrm{~d}$ and $21 \mathrm{~d}$ respectively, and examine the expression of HIF-1 $\alpha$ in hippocampal neurons. Western blot showed significant increases of HIF-1a in all hypobaric hypoxia exposed groups $(p<0.05)$ (Fig. 1A).

Furthermore, immunofluorescence staining of TUNEL/NeuN-positive cells in hippocampus CA3 region showed more apoptotic neurons in the $7 \mathrm{~d}$ and 21d hypobaric hypoxia exposure groups compared with control respectively (Fig. 1B). Besides, western blot for apoptosis related proteins revealed significant higher cleaved caspase-3/caspase-3 and Bax/Bcl-2 ratio in the $7 d$ and $21 d$ exposure groups compared with control $(p<0.01)$ (Fig. 1C). In addition, we observed that the relative intensity of cleaved-caspase- 3 to caspase- 3 in NeuN-positive cells in 21d hypobaric hypoxia exposure group was significantly greater than that of control $(p<0.01)$ (Fig. 1D). Thus, it may be inferred that chronic hypobaric hypoxia exposure leads to neuron apoptosis in hippocampus.

Since it's believed that CIRBP can be induced in hypoxia-related stress, we detected the expression of CIRBP in the process of hypobaric hypoxia exposure. Western blot showed that the CIRBP levels of the $3 \mathrm{~d}$ and $7 \mathrm{~d}$ exposure group were significantly higher than those of control respectively $(p<0.01)$, while the CIRBP level of the 21d exposure group was significantly lower compared with control $(p<0.01)$. It seemed that CIRBP were induced in the early stage of hypoxia exposure and consistently suppressed as the exposure prolonged (Fig. 1E).

\section{Chronic hypobaric hypoxia exposure could induce cell apoptosis and significant decreased CIRBP expression in SH-SY5Y cells}

To examine the function of CIRBP in hypoxia-related neuron apoptosis, we established chronic hypoxia model in vitro. SH-SY5Y cells were cultured in 1\% oxygen concentration for $48 \mathrm{~h}$ in hypoxia chamber to imitate the tissue chronic hypoxic conditions and western blot revealed a significant increase of HIF-1a expression compared with control (Fig. 2A).

Observation under light microscope showed a significant difference of the cell quantity between exposure group and control group (Fig. 2B). Immunofluorescence staining by TUNEL kit observed an increased number of positive cells, while flow cytometry with Annexin V and PI staining counted significantly higher percentage of Annexin $\mathrm{V}^{+} / \mathrm{PI}+$ cells compared with control $(\mathrm{p}<0.01)$ (Fig. 2C/D). In addition, western blot showed significant increases in cleaved caspase-3/caspase- 3 and $\mathrm{Bax} / \mathrm{Bcl} 2$ ratio in comparison with control $(\mathrm{p}<0.01)$ (Fig. 2E). Thus, we inferred that $1 \%$ hypoxia exposure for $48 \mathrm{~h}$ lead to higher ratio of apoptosis in SH-SY5Y cells.

In order to further confirm the role of HIF-1a in chronic hypobaric hypoxia, we established the stable HIF-1a knockdown SH-SY5Y cell line by transfecting the HIF-1a shRNAs and exposed them in $1 \%$ oxygen concentration for $48 \mathrm{~h}$. Flow cytometry with Annexin $\mathrm{V}$ and PI staining showed lower percentage of Annexin V+/ PI + cells in HIF-1a shRNAs cells compared with cell transfected with vehicle control $(p<0.01) \quad$ (Fig. 2F), while western blot showed decreases in cleaved caspase-3/caspase-3 and Bax/Bcl-2 ratio in HIF-1a shRNAs cells $(\mathrm{p}<0.01)$ (Fig. 2G). These results showed that the HIF-1a up-regulation could be pro-apoptotic under chronic hypobaric hypoxia.

CIRBP expression was measured after 1\% hypoxia exposure, and western blot showed that CIRBP expression significant reduced compared with control group, which were consistent with the in vivo results (Fig. 2H).

\section{CIRBP over-expression suppressed HIF-I a up-regulation in hypoxia and inhibited hypoxia-induced neuron apoptosis}

To investigate the potential association between the reduction of CIRBP and hypoxia-induced neuron apoptosis, we examined the effect of CIRBP over-expression on HIF-1a expression and hypoxia-induced apoptosis. We transfected SH-SY5Y cells with p-EGFP-N2-CIRBP plasmid and examined 
the cell apoptosis by flow cytometry and TUNEL kit staining after exposure to $1 \%$ hypoxia for $48 \mathrm{~h}$.
Over-expression of CIRBP in transfected cells was confirmed by Western blot (Fig. 3A).

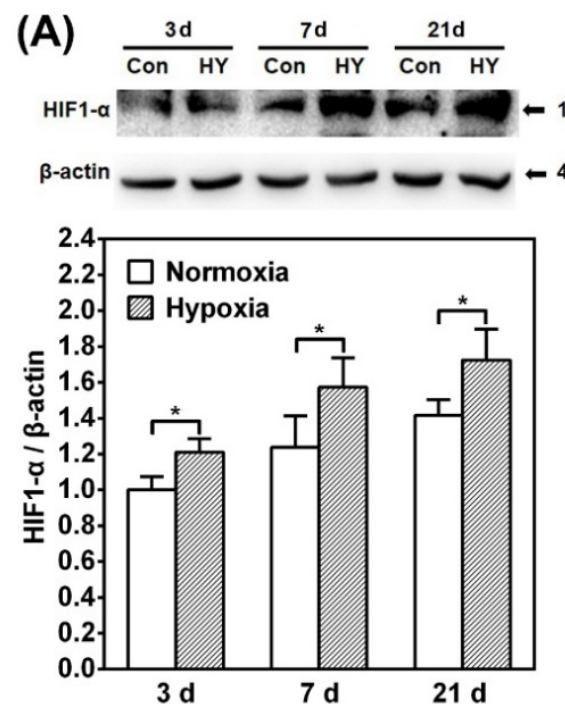

(C)

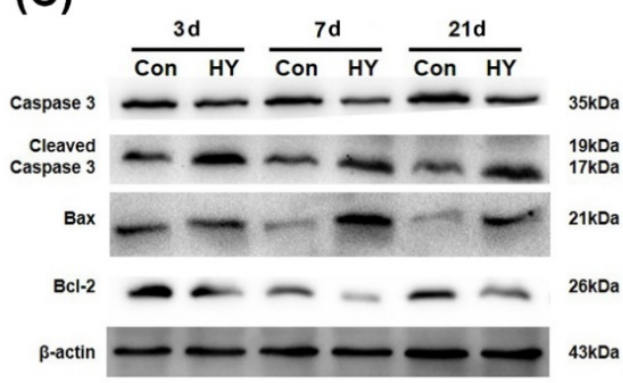

(D) Normoxia
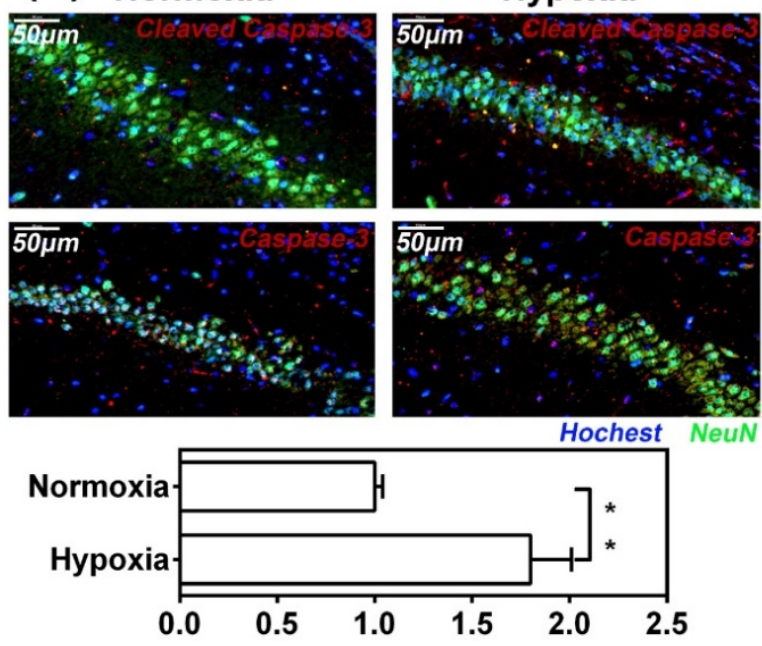

(B)

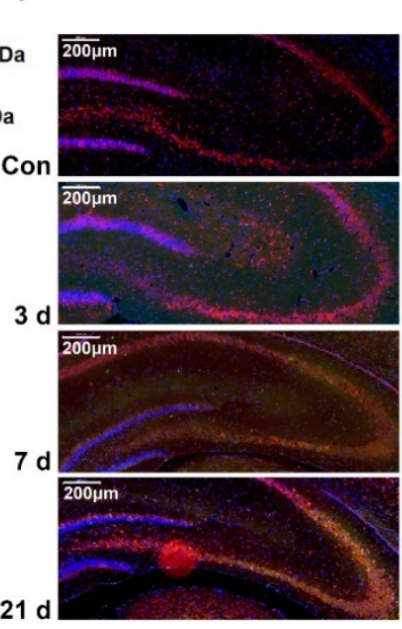

Hochest NeuN TUNEL
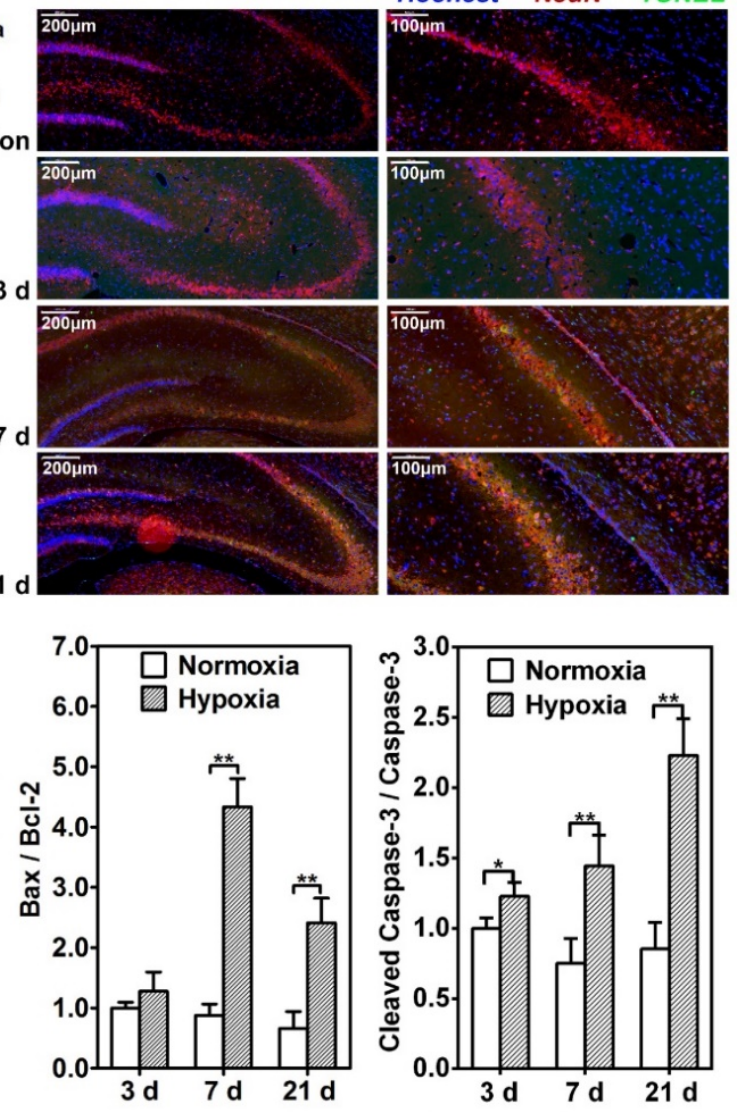

(E)
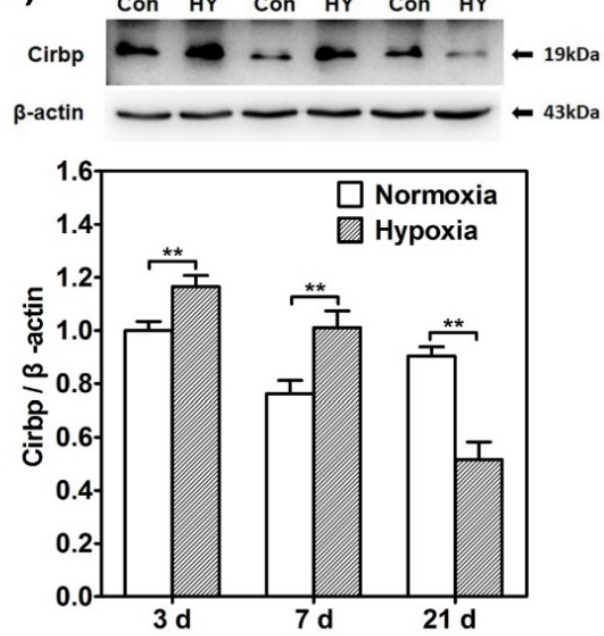

Figure 1. Chronic hypobaric hypoxia exposure led to elevated neuron apoptosis and significant change of CIRBP expression in rat hippocampus. (A) Western blot analysis of HIF-1 $\alpha$ in hippocampus of rats in control groups (normoxia) and hypobaric hypoxia groups continuously for $3 \mathrm{~d}$, $7 \mathrm{~d}$ and $21 \mathrm{~d}$ respectively. Notice the significant increase of HIF-1 $\alpha$ in all hypobaric hypoxia groups compared with control groups. * $(\mathrm{p}<0.05)$. (B) In situ TUNEL apoptosis detection (green) of rat brain tissue samples double stained with $\mathrm{NeuN}$ (red) to distinguish apoptotic neurons in hippocampus after 3d, 7d and $21 \mathrm{~d}$ of hypobaric hypoxia exposure. After 7d and $21 \mathrm{~d}$ of hypobaric hypoxia exposure, more TUNEL/NeuN-positive cells were observed in the hippocampus CA3 region. Scale bar $=200 \mu \mathrm{m}$ (left) and $100 \mu \mathrm{m}$ (right). (C) Western blot analysis of caspase-3, cleaved caspase-3, Bcl-2, Bax and $\beta$-actin in hippocampus of rats kept in control groups or hypobaric hypoxia groups continuously for $3 \mathrm{~d}$, 7d and $21 \mathrm{~d}$ respectively. Graphs show relative ratio of cleaved caspase-3/caspase-3 and Bax/Bcl-2. Notice the significant increase of cleaved caspase-3/ caspased-3 ratio in all hypobaric hypoxia groups and increase of Bax/Bcl-2 ratio in $7 \mathrm{~d}$ and $21 \mathrm{~d}$ hypobaric hypoxia groups compared with the control group, respectively. $*(p<0.05)$, $* *(p<0.01)$. (D) Immunofluorescence staining of cleaved caspase-3 and caspase-3 (red) doubled stained with NeuN (green) respectively in the hippocampus CA3 after 21d of hypobaric hypoxia exposure. Relative intensities of cleaved caspase- 3 were normalized to that of caspase- 3 in NeuN-positive cells. Graphs summarizes the quantitative analysis of analysis. Scale bar $=50 \mu m$. Notice the significant increase of cleaved caspase-3/ caspased-3 intensity in hypoxia group compared with the control group. $* *(p<0.01)$ (E) Western blot analysis of CIRBP in hippocampus of rat kept in control groups or hypobaric hypoxia groups continuously for $3 \mathrm{~d}, 7 \mathrm{~d}$ and $21 \mathrm{~d}$ respectively. There was a significant increase in CIRBP in $3 \mathrm{~d}$ and $7 \mathrm{~d}$ hypobaric hypoxia groups, and a significant decrease of CIRBP in $21 \mathrm{~d}$ hypobaric hypoxia group compared with the control group. ** $(\mathrm{p}<0.01)$. 


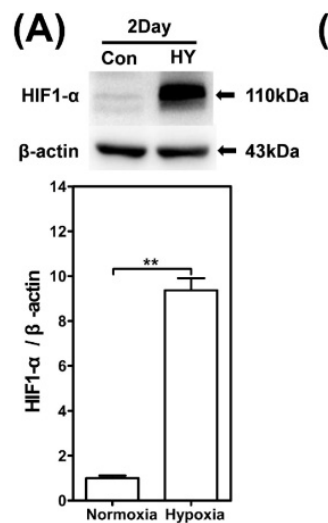

(D)

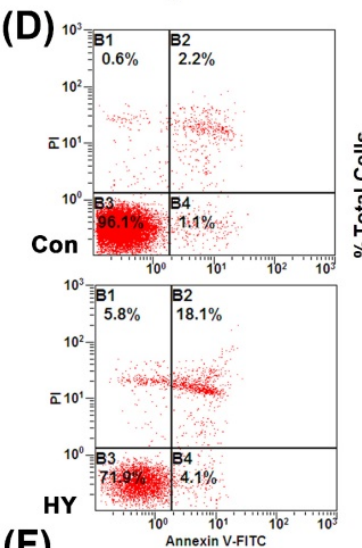

(F)

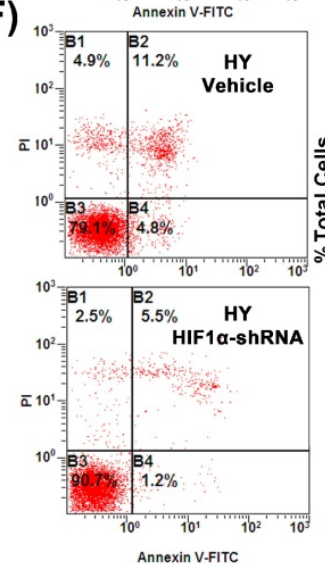

(B)
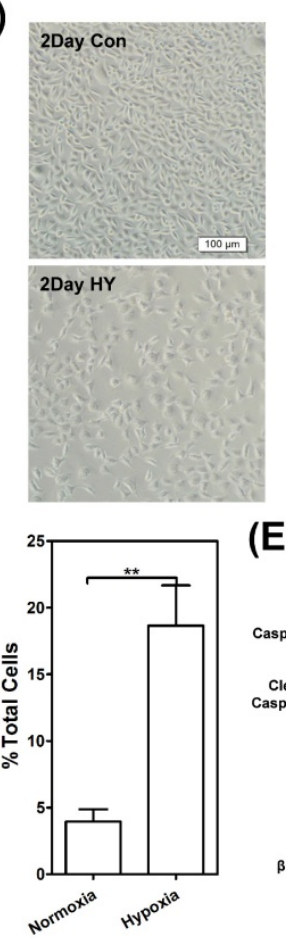

(E)

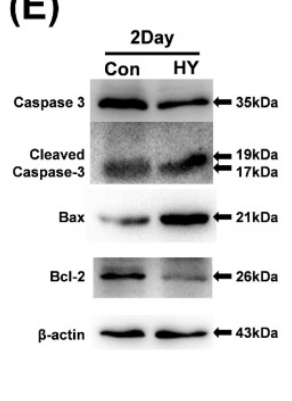

(G)

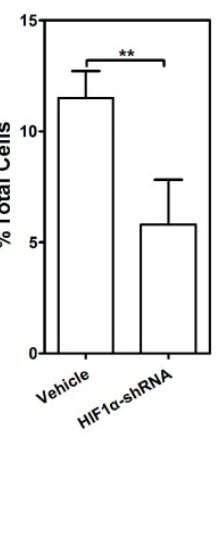

(C)

\section{(G)}
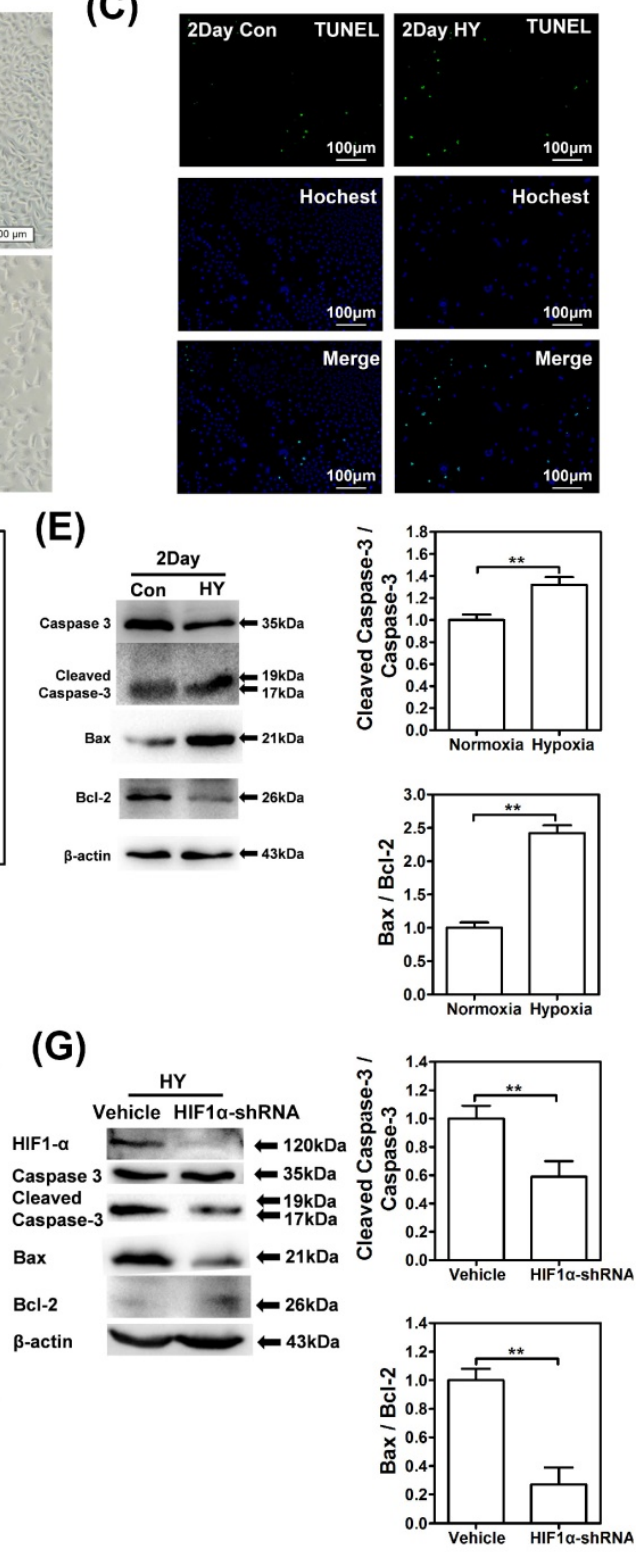

(H)

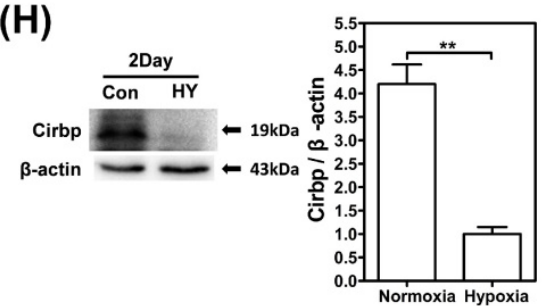

Figure 2. Chronic hypobaric hypoxia exposure could induce cell apoptosis and significant decreased CIRBP expression in SH-SY5Y cells. (A) Western blot analysis of HIF-1 $\alpha$ in in SH-SY5Y cells cultured under normoxia or $1 \%$ hypoxia for 48 hours. Notice the significant increase of HIF-1 $\alpha$ in the hypoxia group compared with control group. ** $(\mathrm{p}<0.01)$. (B) Photomicrographs of SH-SY5Y cells cultured under normoxia or hypoxia for 48 hours. There was a decrease of cell density under light microscope in the hypoxia group compared with the control group. (C) TUNEL apoptosis detection of SH-SY5Y cells cultured under normoxia or $1 \%$ hypoxia for 48 hours. Increased TUNEL-positive cells were observed in hypoxia group compared with the control group. Bars $=100 \mu \mathrm{m}$. (D) Flow cytometry shows apoptosis of SH-SY5Y cells by Annexin V and PI staining in control or hypoxia group for 48 hours. $1 * 10^{6}$ cell were counted for each group. B1 necrotic cells; B2 late apoptotic cells; B3 viable cells; B4 early apoptosis. Bar graphs demonstrating higher ratio of apoptotic cells in hypoxia group compared with control. $* *(\mathrm{p}<0.01$ ). (E) Western blot analysis of caspase-3, cleaved caspase-3, Bcl-2, Bax and $\beta$-actin in SH-SY5Y cells cultured under normoxia or $1 \%$ hypoxia for 48 hours. Graphs show relative ratio of cleaved caspase-3/caspase-3 and Bax/Bcl-2. Notice the significant increase of cleaved caspase-3/caspase-3 and Bax/Bcl-2 ratio in the hypoxia group compared with the control group, respectively. $* *(P<0.01)$. $(\mathrm{F})$ Flow cytometry shows apoptosis of SH-SY5Y cells transfected with HIF-1 a shRNAs and vehicle control exposed in 1\% oxygen concentration for 48h. Bar graphs demonstrating lower ratio of apoptotic cells transfected with HIF-1a shRNAs compared with cells transfected with vehicle control. ** $(\mathrm{p}<0.01)$. (G) Western blot analysis of HIF-1 1 , caspase-3, cleaved caspase-3, Bcl-2, Bax and $\beta$-actin in SH-SYSY cells transfected with HIF-la shRNAs and vehicle control exposed in $1 \%$ oxygen concentration for $48 \mathrm{~h}$. Graphs show relative ratio of cleaved caspase-3/caspase-3 and Bax/Bcl-2. Notice the decrease of cleaved caspase-3/caspase-3 and Bax/Bcl-2 ratio in HIF-1 $\alpha$ shRNAs transfected cells compared with vehicle transfected cells respectively. ** $(\mathrm{p}<0.01)$. (H) Western blot analysis of CIRBP expression in SH-SY5Y cells cultured under normoxia or hypoxia for 48 hours. There was significant decrease of CIRBP expression in the hypoxia group compared with the normoxia group. $* *(P<0.01)$. 


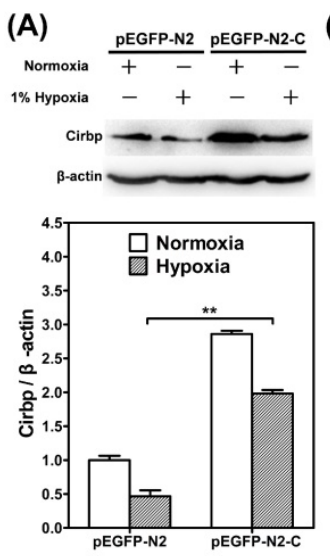

(D)
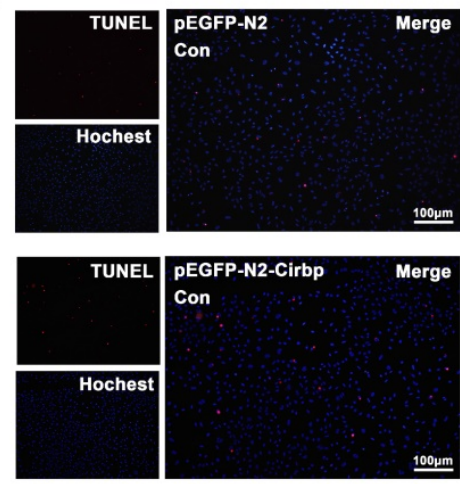

(B)

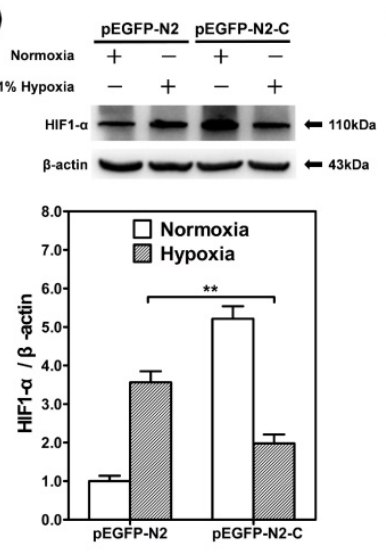

C)
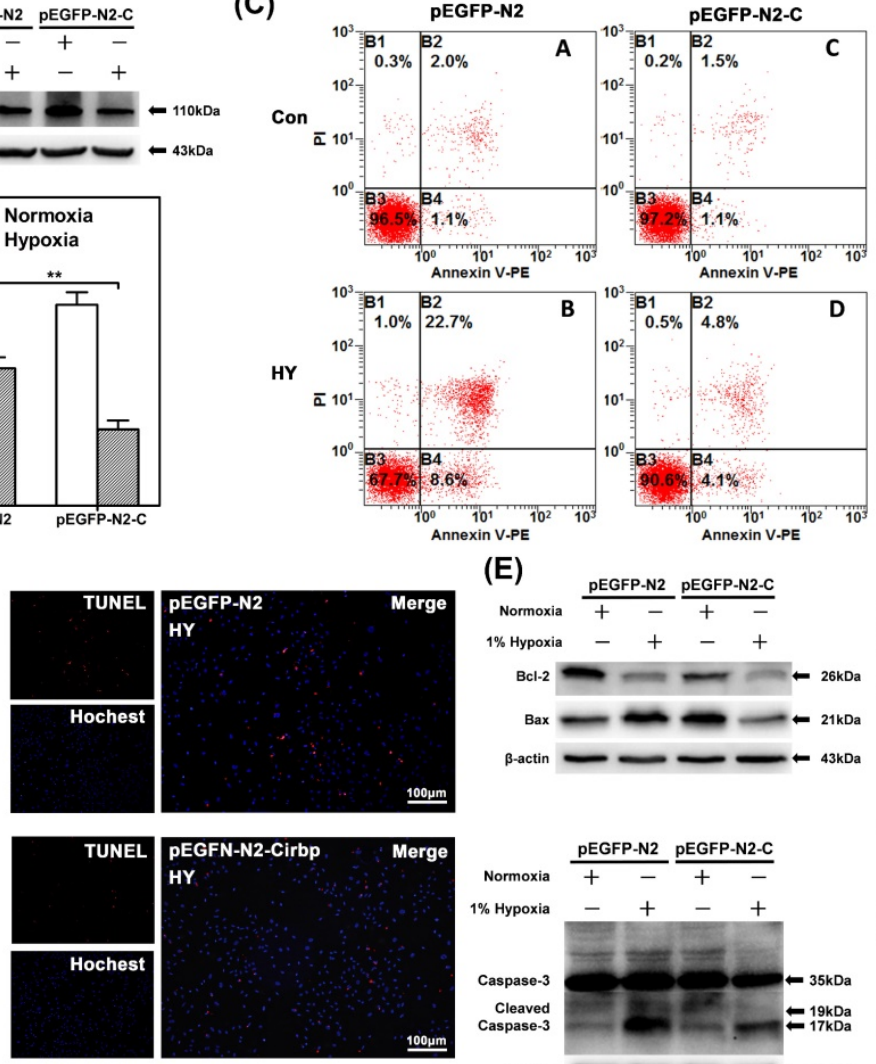

(E)
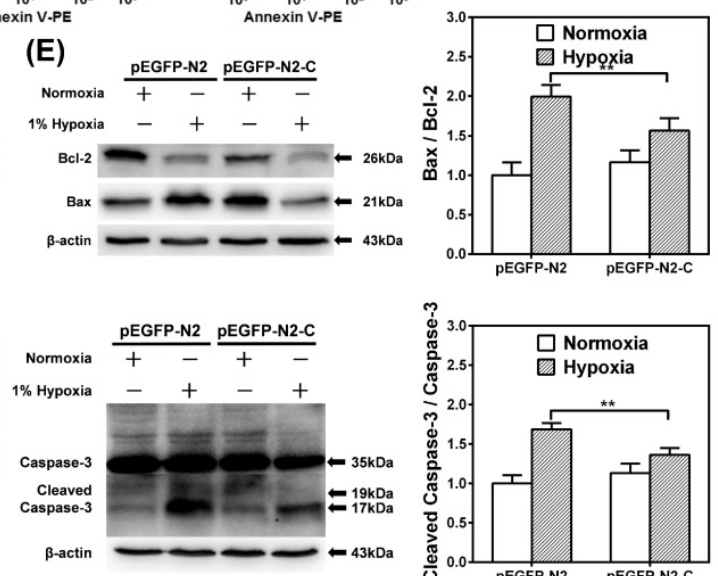
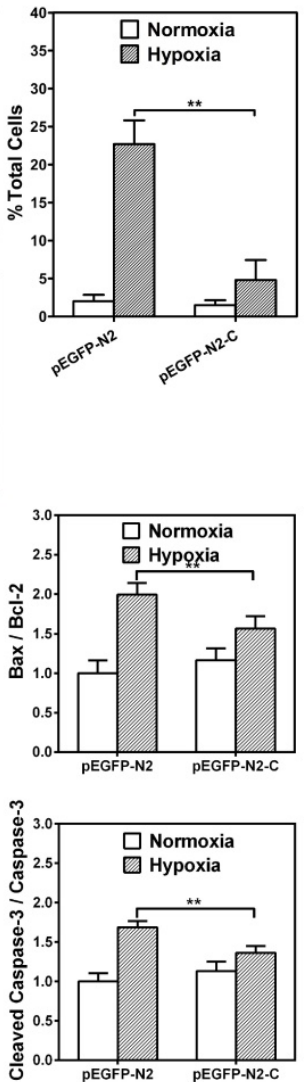

Figure 3. CIRBP over-expression suppressed HIF-1 $\alpha$ up-regulation in hypoxia and inhibited hypoxia-induced neuron apoptosis. Western blot analysis of CIRBP expression levels (A) and HIF-1 $\alpha$ (B) in SH-SY5Y cells transfected with mock vector (pEGFP-N2) or pEGFP-N2-CIRBP (pEGFP-N2-C) cultured in normoxia or 1\% hypoxia for $48 \mathrm{~h}$. ** depicts the significant increase of CIRBP and decrease of HIF-1 $\alpha$ transfected with pEGFP-N2-C in hypoxia group compared with control group. (C) Flow cytometry shows apoptosis of SH-SY5Y cells transfected with mock vector (pEGFP-N2) or pEGFP-N2-CIRBP (pEGFP-N2-C) cells by Annexin V and PI staining in control or hypoxia group for 48 hours. Bar graphs demonstrating extent of apoptotic cells, ** depicts the significant decrease of ratio of apoptotic cells in cells transfected with pEGFP-N2-CIRBP in hypoxia group compared with cells in control group. (D) TUNEL apoptosis detection of SH-SY5Y neuron-like cells transfected with pEGFP-N2 or pEGFP-N2-CIRBP cultured under normoxia or hypoxia for 48 hours. Decrease TUNEL-positive cells were observed in cells transfected with pEGFP-N2-CIRBP in hypoxia group compared with control groups. Bars $=100 \mu \mathrm{m}$. (E) Western blot analysis of caspase-3, cleaved caspase-3, Bcl-2, Bax and $\beta$-actin in SH-SY5Y neuron-like cells transfected with pEGFP-N2 or PEGFP-N2-CIRBP in control group or hypoxia. Graphs show relative ratio of cleaved caspase-3/caspase-3 and Bax/Bcl-2. Notice the significant decrease in cleaved caspase-3 and $\mathrm{Bax} / \mathrm{Bcl}-2$ ratio in cells transfected with PEGFP-N2-CIRBP in the hypoxia groups compared with the control group, respectively. $* *(\mathrm{P}<0.01)$.

CIRBP over-expression significantly decreased the levels of HIF-1a under $1 \%$ hypoxia $(\mathrm{p}<0.01)$ (Fig. 3B). Under normoxic culture condition, there were no significant differences of the percentages of Annexin $\mathrm{V}^{+} / \mathrm{PI}+$ cells between mock vector and p-EGFP-N2-CIRBP-transfected cells. However, under $1 \%$ hypoxia, over-expression of CIRBP decreased the percentages of Annexin $\mathrm{V}^{+} / \mathrm{PI}+$ cells $(p<0.01)$ (Fig. 3C). TUNEL kit staining showed similar result that over-expression of CIRBP significantly decreased the number of TUNEL positive cells under $1 \%$ hypoxia (Fig. 3D). As for apoptosis-related proteins, CIRBP over-expression significantly alleviated the elevated cleaved caspase-3/caspase- 3 and $\mathrm{Bax} / \mathrm{Bcl} 2$ ratio under $1 \%$ hypoxia $(\mathrm{p}<0.01)$ (Fig. 3E). Taken together, these results suggested that over-expression of CIRBP could alleviate the hypoxia-induced apoptosis in SH-SY5Y cells through regulating in a HIF-1a expression.

\section{MiR-23a could up-regulate CIRBP and down-regulate HIF-1 $\alpha$ under $1 \%$ hypoxia}

In order to identify the potential regulating function of hypoxia-related miRNAs in chronic hypobaric hypoxia induced apoptosis, we aimed at miR-1 and miR-23a and measured their expression in vivo and in vitro. As shown in Fig. $\mathbf{4 A / B}$, in the $3 \mathrm{~d}$ hypoxia exposed rats, miR-23a level was significantly higher compared with control, however in $7 \mathrm{~d} / 21 \mathrm{~d}$ hypoxia exposed rats, miR-23a levels were significantly lower than those of control $(p<0.01)$. Similarly, in the $3 \mathrm{~d} / 7 \mathrm{~d}$ hypoxia exposed rats, miR-1 levels were significantly higher compared with control, but in 21d exposed rats, miR-1 level was significantly lower than those of control $(p<0.01)$.

We further established miR-23a/miR-1 inhibitor and mimic plasmid (Fig. 4C/D) and assessed whether down-regulating/up-regulating of miR-23a/miR-1 affected the CIRBP expression under hypoxia. 
Western blot was performed to compare the expression of CIRBP in SH-SY5Y cells transfected with miR-1 and miR-23a inhibitors and mimics respectively. As for miR-23a, transfected inhibitors significantly decreased the CIRBP expression in SH-SY5Y cells under hypoxia $(p<0.01)$, while transfected mimics significantly increased CIRBP expression $(p<0.01)$. Furthermore, we found miR-23a inhibitor increased HIF-1a expression $(p<0.01)$ while miR-23a mimics decreased HIF-1a expression $(p<0.01)$. (Fig. 4E) However, as for miR-1, there were no differences of CIRBP and HIF-1a expression between the cells transfected with inhibitors and those with mimics. These results indicated that miR-23a up-regulated CIRBP and down-regulated HIF-1a under $1 \%$ hypoxia. (Fig. 4F)

\section{MiR-23a was involved in the regulation of hypoxia-induced neuron apoptosis}

To further confirm the function of miR-23a in hypoxia induced neuron apoptosis, we transfected SH-SY5Y cells with miR-23a inhibitors and mimics, and assessed the apoptosis with flow cytometry. Under $1 \%$ hypoxia exposure, transfected miR-23a inhibitors significantly increased the percentages of cells in early apoptosis phase $(p<0.01)$, while transfected mimics significantly reduced the percentages of cells in early apoptosis phase $(p<0.01)$.While, under normoxic culture condition, neither of transfected miR-23a inhibitor/mimic could affect the percentage of early apoptosis cells. (Fig. 5A)

Furthermore, the expression of apoptosis related proteins under $1 \%$ hypoxia was also affected by the change of miR-23a level. Transfected miR-23a inhibitors significantly enhanced cleaved caspase-3/caspase-3 and Bax/Bcl-2 ratio $(p<0.01)$, while transfected mimics significantly reduced the expression of the proteins $(\mathrm{p}<0.01)$. Under normoxic culture condition, the change of miR-23a did not affect the expression of apoptosis related proteins (Fig. 5B).

However, as for miR-1, there were no differences of cell apoptosis under hypoxia between the cells transfected with inhibitors and those with mimics (Fig. 5C). Taken together, these results suggested that miR-23a was involved in the regulation of hypoxia-induced cell apoptosis.
(A)

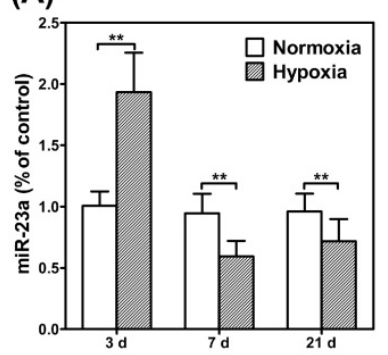

(B)

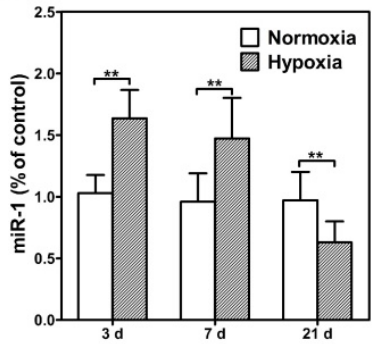

(E)
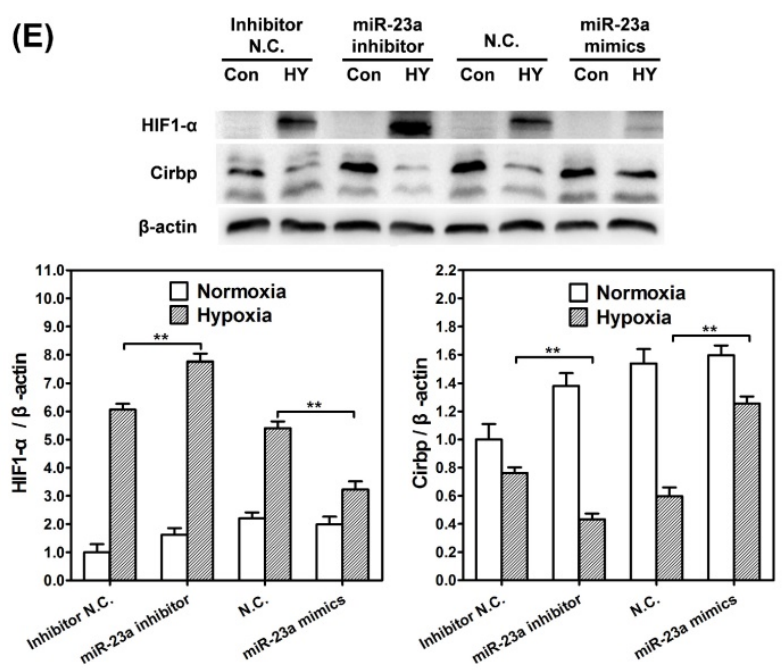

(C)

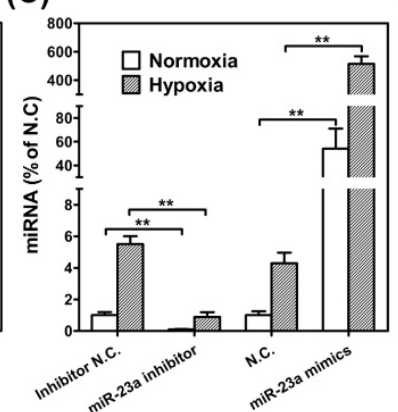

(D)

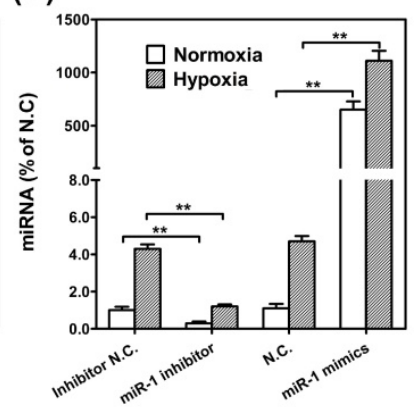

(F)
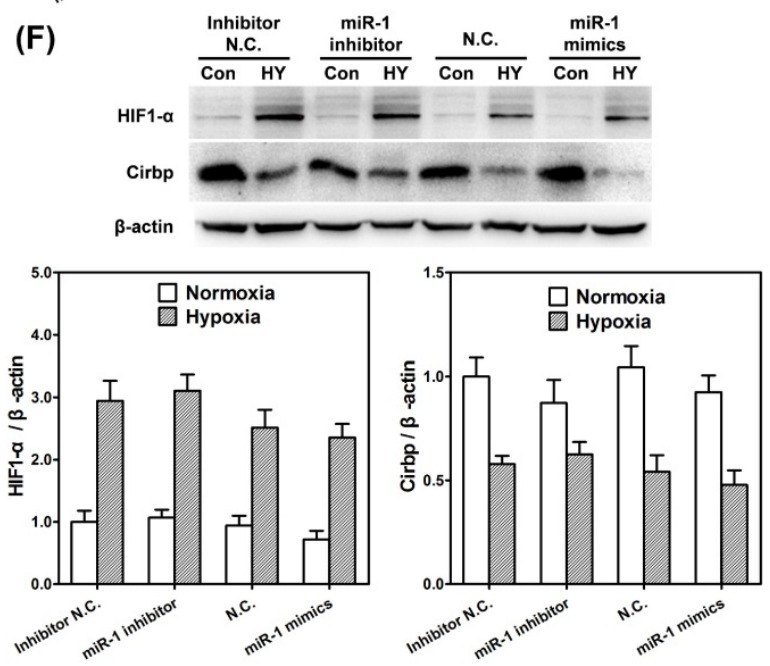

Figure 4. MiR-23a could up-regulate CIRBP and down-regulate HIF-1 $\alpha$ under $1 \%$ hypoxia. Real time RT-PCR of miR-23a (A) and miR-1 (B) in hippocampus of rat kept in control groups or hypobaric hypoxia groups continuously for $3 \mathrm{~d}, 7 \mathrm{~d}$ and $21 \mathrm{~d}$ respectively. There was significant increase in miR-23a in the $3 \mathrm{~d}$ hypobaric hypoxia group, and a significant decrease in miR-23a in the $7 \mathrm{~d}$ and $21 \mathrm{~d}$ hypoxia exposure groups compared with the control group. ** ( $P<0.01)$. For miR-1, there was a significant increase in the $3 \mathrm{~d}$ and $7 \mathrm{~d}$ hypoxia groups and a significant decrease in the $21 \mathrm{~d}$ hypoxia group compared with the control group. RT-PCR of miR-23a expression(C) or miR-1 expression (D) in SH-SY5Y cells transfected with inhibitors and mimics cultured in normoxia and 1\% hypoxia, respectively. Western blot analysis of CIRBP expression and HIF-1a in SH-SY5Y cells transfected with miR-23a inhibitors and mimics $(\mathrm{E})$ or cells transfected with miR-1 inhibitors and mimics $(\mathrm{F})$. $* *(\mathrm{P}<0.01)$. 

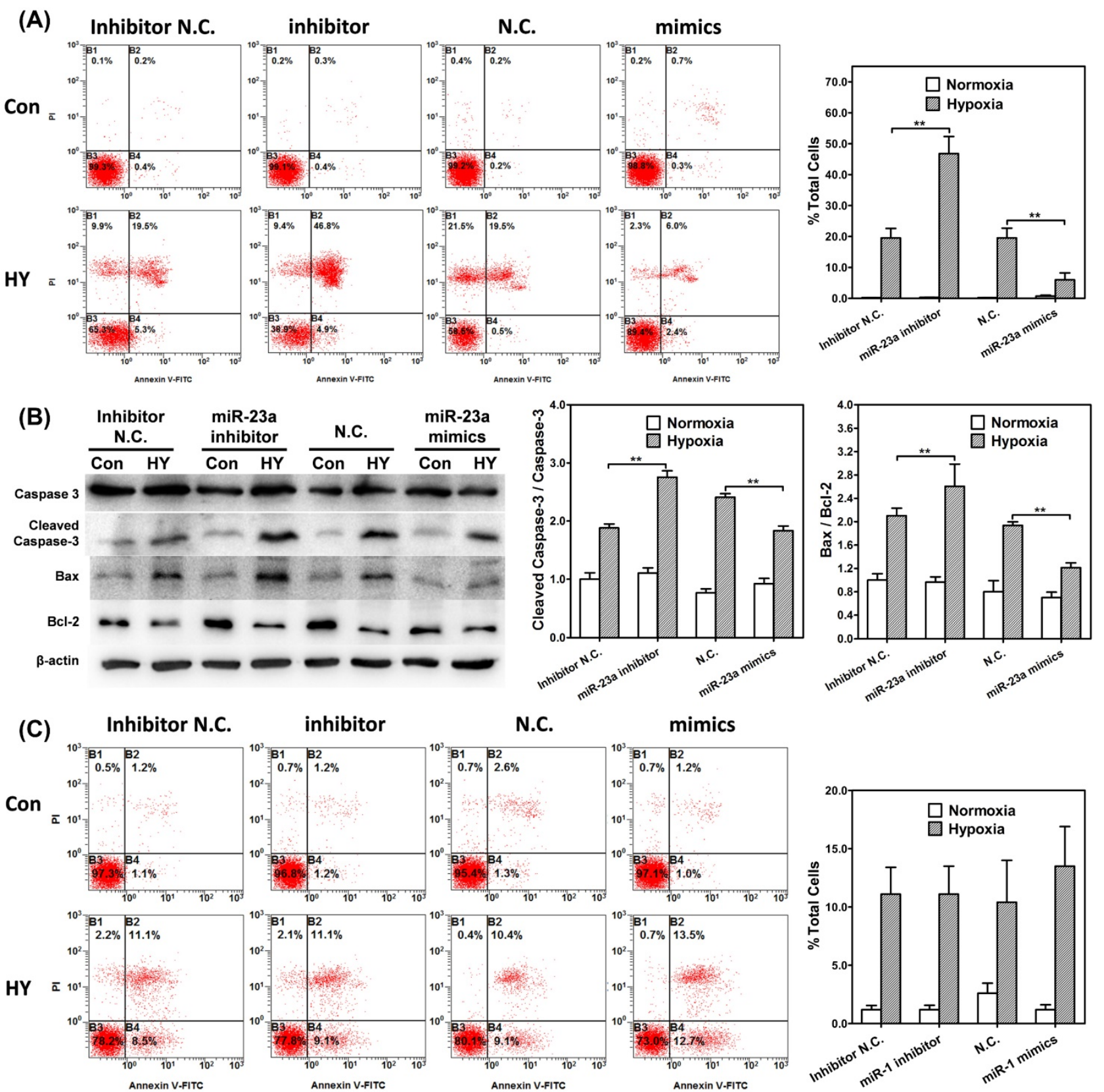

Figure 5. MiR-23a was involved in the regulation of hypoxia-induced neuron apoptosis. Flow cytometry shows apoptosis of SH-SY5Y cells transfected with miR-23a (A) inhibitors and mimics by Annexin $\mathrm{V}$ and PI staining in control or hypoxia group for 48 hours. Bar graphs demonstrating extent of apoptotic cells, notice the significant change of ration of apoptotic cells in cells transfected with miR-23a inhibitors or mimics in hypoxia group compared with cells in control group. ** ( $P<0.01$ ). (B) Western blot analysis of caspase-3, cleaved caspase-3, Bcl-2, Bax and $\beta$-actin in SH-SY5Y neuron-like cells transfected with miR-23a inhibitors and mimics cultured in normoxia or hypoxia for 48 hours. Graphs show relative ratio of cleaved caspase-3/caspase-3 and Bax/Bcl-2.** ( $\mathrm{p}<0.01$ ). (C) Flow cytometry shows apoptosis of SH-SY5Y cells transfected with miR-1 inhibitors and mimics by Annexin V and PI staining in control or hypoxia group for 48 hours. Bar graphs demonstrating extent of apoptotic cells. There was no difference of apoptosis ratio in hypoxic exposed between the cells transfected with miR-1 inhibitors/mimics and those transfected with negative control.

\section{Discussion}

The study was to investigate the molecular mechanisms by which CIRBP involved in cell apoptosis during chronic hypobaric hypoxia stress. We found that CIRBP expression was down-regulated in both rat hippocampal neurons and SH-SY5Y cells exposed to hypoxia. In addition, over-expressing CIRBP could efficiently suppress HIF-1a up-regulation and thus inhibit hypoxia induced neuron apoptosis. Furthermore, by screening hypoxia-related miRNAs in hypoxia models, we observed up-regulated miR-23a after hypoxia exposure, which may be a potential target for maintaining the CIRBP expression in chronic hypobaric hypoxia and reducing neuron apoptosis.

Hypobaric hypoxia has a wide spectrum of pathophysiological effects on human body, especially the brain. Previous studies have assessed the dynamic changes in peripheral and cerebral oxygenation 
engendered by ascent to altitude $4000 \mathrm{~m}$ and found that peripheral oxygenation decreased in the 2 nd day of exposure and then partially rebounded in the 7 th day $[42,45,46]$. In this study, we measured HIF-1a, a well-known cellular hypoxia indicator, in order to reveal the oxygenation condition of local cells in rat hippocampus. We found since the 7 th day of exposure, HIF-1a expression in hypoxia group had been significantly higher than that of control, suggesting that chronic hypobaric hypoxia exposure could cause consistent low oxygen content within hippocampal neurons. As previously reported, apoptosis can be induced in response to hypoxia and the severity of hypoxia determines whether cells become apoptotic or adapt to hypoxia and survive [9]. Previous studies have reported pyramidal neurons damage under chronic hypoxia [6,7]. Similarly, in this study, we found higher ratio of neuron apoptosis in the hippocampus CA3 region of hypoxia exposed rats compared with control, indicating chronic hypobaric hypoxia may lead to substantial neural injuries. Besides, elevated HIF-1a and ratio of neuron apoptosis were also confirmed in $1 \%$ hypoxia exposed SH-SY5Y cells. Notably, knocking down HIF-1a by transfecting the HIF-1a shRNAs lead to significant lower percentage of Annexin V+/ PI + cells and decreased cleaved caspase-3/caspase-3 and Bax/Bcl-2 ratio in SH-SY5Y cells, suggesting that HIF-1a up-regulation should be pro-apoptotic in chronic hypobaric hypoxia in this study.

CIRBP has been implicated in neural apoptosis induced by various environmental stress, such as hypothermia, oxidative stress, inflammation and DNA damage [47-49]. In moderate low temperature, CIRBP could be induced in both neurons and neural stem cells and exerts neuroprotective effect [48]. In inflammation [49-51], overexpressing CIRBP could protect cells from TNF-a-induced apoptosis by activating extracellular signal-regulated kinase (ERK) pathways in CIRBP-deficient mouse fibroblasts. What's more, in rat cortical neurons, CIRBP induction could inhibit etoposide-induced apoptosis by regulating levels of p53 and its downstream targets [47]. The role of CIRBP under chronic hypobaric hypoxia remains less mentioned. In this study, we found that in the hippocampus of chronic hypobaric hypoxia exposed rats, CIRBP expression increased in the early stage of exposure, and then decreased and kept consistently suppressed after the 7th day. Correspondingly, both the ratio of neuron apoptosis in hippocampus CA3 region and apoptosis related proteins increased since the 7th day of exposure. According to the $1 \%$ hypoxia exposed SH-SY5Y cells, CIRBP expression increased in the first $12 \mathrm{~h}$ of exposure and then decreased and kept consistently suppressed after $24 \mathrm{~h}$ of hypoxia exposure. Thus, it could be speculated that down-regulation of CIRBP may be involved in chronic hypobaric hypoxia induced neuron apoptosis.

HIF-1a was the most important transcription factor in cellular hypoxia response, whose role in hypoxia induced apoptosis has been widely discussed $[9,16,17,52]$. HIF-1a could initiate hypoxia mediated apoptosis by increasing the expression of Bcl-2 binding proteins thereby inhibiting the anti-apoptotic effect of Bcl-2 [11, 12, 15]. The association between CIRBP and HIF-1a in hypoxia has been discussed, but there is no unified conclusion reached. Elizabeth T. Chang found that CIRBP could bind to HIF-1a and several protein translation factor mRNAs on polysomes and increase de novo protein translation under cellular stress [30]. In this study, CIRBP over-expression significantly decreased the level of HIF-1a and the ratio of apoptosis in the $1 \%$ hypoxia exposed SH-SY5Y cells, indicating that over-expressing CIRBP could suppress HIF-1a expression and alleviate the hypoxia induced apoptosis. Recently, Weibo Luo et al. reported several HIF repressors under chronic hypoxia [53]. They found several genes, such as peroxiredoxin 2 (PRDX2) and PRDX4, suppressed the level of HIF-1a mRNA and its transcriptional activity. We speculate that CIRBP may as a HIF-1a repressor in the process of chronic hypobaric hypoxia induced neuron apoptosis.

Interestingly, compared to that under hypoxia, we found transfection of CIRBP robustly increased HIF-1a expression under normoxia. According to previous studies, HIF-1a has been proved to accumulate under normoxia in several previous studies, and this phenomenon is poorly understood [54-58]. Matthias Kappler et al. demonstrated that normoxic accumulation of HIF-1a may be associated with increased glycolysis or glutaminolysis [56]. Since CIRBP has been proved to be widely involved in cell metabolism, it could be inferred that under normoxia CIRBP transfection may change the cell metabolism and thus lead to HIF-1a accumulation. Besides, HIFs are primarily regulated by the changes in protein stability in an oxygen-dependent manner. Under normoxia, HIF-1a may be immediately proteasomal degraded and thus have little function [16]. In our analysis, flow cytometry apoptosis analysis showed that under normoxia there was no difference between the apoptosis ratio of the CIRBP transfected cells and that of control, and so was the levels of the apoptosis related proteins. Herein, although CIRBP transfection robustly increased HIF-1a accumulation under normoxia, it seemed to have no effect on the apoptosis of SH-SY5Y cells. The detailed mechanism by which CIRBP boosted HIF-1a under normoxia may be 
discussed in the future studies.

MiRNAs function in RNA silencing and post-transcriptional regulation of gene expression [59]. Hypoxia-related miRNAs, including miR-23a, miR-1 and miR-303, were measured in both in vivo and in vitro model. We found miR-23a and miR-1 was induced in both models. Previous studies reported that miR-23a were related to p53 functional status, caspase- 3 activity and cell apoptosis [60]. In tongue squamous cell carcinoma cells, miR-23a could promote cisplatin chemoresistance and alleviate cisplatin-induced apoptosis [61]. However, the roles of miR-23a and its target gene in neurons were not well understood. In in vivo model, we found that in the $3^{\text {rd }}$ and $7^{\text {th }}$ day of exposure, miR-23a levels of the hypoxia group were significantly higher than those of control. However, as the exposure prolonged, miR-23a level of hypoxia group reduced and in the 21th day of exposure, it was significantly lower than that of control. Similarly, in in vitro model, the miR-23a level showed the same tendency. Interestingly, in both models, the miR23a level had been changing in the same pattern (slightly in advance) with CIRBP. Herein, we guess miR-23a may be involved in CIRBP regulation in the process of hypoxia induced apoptosis. Furthermore, we found that miR-23a inhibitors significantly reduced CIRBP expression and suppressed cell apoptosis, while miR-23a mimics enhanced CIRBP expression and decreased cell apoptosis in vitro models, further validating that miR-23a might be a regulator for CIRBP. As for miR-1, previous studies have showed that its expression increased in the first 6 hours of oxygen/glucose deprivation in neuro-2a cells and it could effectively attenuate the expression of heat shock protein HSP-70 mRNA and protein expressions, reduce apoptosis via the intrinsic Bax-mitochondrion-caspase protease pathway [36, 62]. However, in our analysis, although miR-1 was induced in chronic hypoxia, over-expressing/knocking-down miR-1 did not change the CRIBP expression and apoptosis ratio. It suggested that hypoxia-induced miR-1 might only function in the acute stage instead of chronic stage of hypoxia, and it seemed not involved in the regulating of CRIBP expression.

In summary, this study showed that chronic hypobaric hypoxia exposure caused hypoxia insults in rat hippocampus and lead to neuron apoptosis. In parallel, 1\% hypoxia exposure augmented HIF-1a, apoptosis related proteins levels and increased ratio of apoptosis in SH-SY5Y cells. CIRBP, which was induced by hypoxia, were thought to be neuroprotective under chronic hypobaric hypoxia stress. Over-expressing CIRBP could efficiently suppress the HIF-1a expression in cells and thus attenuate hypoxia induced apoptosis. However, CIRBP expression gradually decreased as the exposure prolonged. By screening the hypoxia-related miRNAs, we found that miR-23a was induced by chronic hypobaric hypoxia stress and interestingly its expression changes under exposure shared the similar pattern with CIRBP, indicating that miR-23a might be a potential target for maintaining CIRBP expression in chronic hypobaric hypoxia exposure and reducing neuron apoptosis.

\section{Acknowledgement}

This work was supported by grants from National Natural Science Foundation of China (\#81330045, \#8150277).

\section{Competing Interests}

The authors have declared that no competing interest exists.

\section{References}

1. Xu L, Wu Y, Zhao T, Liu SH, Zhu LL, Fan M, et al. [Effect of high altitude hypoxia on cognitive flexibility]. Zhongguo ying yong sheng li xue za zhi = Zhongguo yingyong shenglixue zazhi $=$ Chinese journal of applied physiology. 2014; 30: 106-9, 18.

2. Lieberman P, Morey A, Hochstadt J, Larson M, Mather S. Mount Everest: a space analogue for speech monitoring of cognitive deficits and stress. Aviation, space, and environmental medicine. 2005; 76: B198-207.

3. Bartholomew CJ, Jensen W, Petros TV, Ferraro FR, Fire KM, Biberdorf D, et al. The effect of moderate levels of simulated altitude on sustained cognitive performance. The International journal of aviation psychology. 1999; 9: 351-9.

4. Shukitt-Hale B, Stillman MJ, Welch DI, Levy A, Devine JA, Lieberman HR. Hypobaric hypoxia impairs spatial memory in an elevation-dependent fashion. Behavioral and neural biology. 1994; 62: 244-52.

5. Iwasaki K, Zhang R, Zuckerman JH, Ogawa Y, Hansen LH, Levine BD. Impaired dynamic cerebral autoregulation at extreme high altitude even after acclimatization. Journal of cerebral blood flow and metabolism : official journal of the International Society of Cerebral Blood Flow and Metabolism. 2011; 31: 283-92.

6. Maiti P, Singh SB, Muthuraju S, Veleri S, Ilavazhagan G. Hypobaric hypoxia damages the hippocampal pyramidal neurons in the rat brain. Brain research. 2007; 1175: 1-9.

7. Shukitt-Hale B, Kadar T, Marlowe BE, Stillman MJ, Galli RL, Levy A, et al. Morphological alterations in the hippocampus following hypobaric hypoxia. Human \& experimental toxicology. 1996; 15: 312-9.

8. Ueno M, Tomita S, Ueki M, Iwanaga Y, Huang CL, Onodera M, et al. Two pathways of apoptosis are simultaneously induced in the embryonal brains of neural cell-specific HIF-1alpha-deficient mice. Histochemistry and cell biology. 2006; 125: 535-44.

9. Li L, Qu Y, Mao M. [Relationship between hypoxia inducible factor 1alpha: expression and neuron apoptosis during hypoxia ischemia brain damage in neonatal rats]. Zhongguo xiu fu chong jian wai ke za $\mathrm{zhi}=$ Zhongguo xiufu chongjian waike zazhi $=$ Chinese journal of reparative and reconstructive surgery. 2007; 21: 1326-9.

10. Sarada SK, Himadri P, Ruma D, Sharma SK, Pauline T, Mrinalini. Selenium protects the hypoxia induced apoptosis in neuroblastoma cells through upregulation of Bcl-2. Brain research. 2008; 1209: 29-39.

11. Sermeus A, Genin M, Maincent A, Fransolet M, Notte A, Leclere L, et al. Hypoxia-induced modulation of apoptosis and BCL-2 family proteins in different cancer cell types. PloS one. 2012; 7: e47519.

12. Wu $\mathrm{XY}$, Hao $\mathrm{CP}$, Ling $\mathrm{M}$, Guo $\mathrm{CH}$, Ma W. Hypoxia-induced apoptosis is blocked by adrenomedullin via upregulation of Bcl-2 in human osteosarcoma cells. Oncology reports. 2015; 34: 787-94.

13. Janke K, Brockmeier U, Kuhlmann K, Eisenacher M, Nolde J, Meyer HE, et al. Factor inhibiting HIF-1 (FIH-1) modulates protein interactions of apoptosis-stimulating p53 binding protein 2 (ASPP2). Journal of cell science. 2013; 126: 2629-40.

14. Suzuki H, Tomida A, Tsuruo T. Dephosphorylated hypoxia-inducible factor 1alpha as a mediator of p53-dependent apoptosis during hypoxia. Oncogene. 2001; 20: 5779-88. 
15. Yin R, Yuan L, Ping L, Hu L. Neonatal bronchopulmonary dysplasia increases neuronal apoptosis in the hippocampus through the HIF-1alpha and p53 pathways. Respiratory physiology \& neurobiology. 2016; 220: 81-7.

16. Fu Z, Chen D, Cheng H, Wang F. Hypoxia-inducible factor-1alpha protects cervical carcinoma cells from apoptosis induced by radiation via modulation of vascular endothelial growth factor and p53 under hypoxia. Medical science monitor : international medical journal of experimental and clinical research. 2015; 21: 318-25

17. Sendoel A, Kohler I, Fellmann C, Lowe SW, Hengartner MO. HIF-1 antagonizes p53-mediated apoptosis through a secreted neuronal tyrosinase. Nature. 2010; 465: 577-83.

18. Pan F, Zarate J, Choudhury A, Rupprecht R, Bradley TM. Osmotic stress of salmon stimulates upregulation of a cold inducible RNA binding protein (CIRP) similar to that of mammals and amphibians. Biochimie. 2004; 86: 451-61

19. Nishiyama H, Higashitsuji $H$, Yokoi H, Itoh K, Danno S, Matsuda T, et al. Cloning and characterization of human CIRP (cold-inducible RNA-binding protein) cDNA and chromosomal assignment of the gene. Gene. 1997; 204: 115-20.

20. Li SZ, Jin FH, Zhao QX. [Progress of research on CIRP and its biological functions]. Sheng li ke xue jin zhan [Progress in physiology]. 2009; 40: 271-3.

21. Tang JJ, Tang C, Nie PT. [The cytoprotective mechanisms of CIRP upon stresses]. Sheng li ke xue jin zhan [Progress in physiology]. 2013; 44: 67-71.

22. Yang C, Carrier F. The UV-inducible RNA-binding protein A18 (A18 hnRNP) plays a protective role in the genotoxic stress response. The Journal of biological chemistry. 2001; 276: 47277-84

23. De Leeuw F, Zhang T, Wauquier C, Huez G, Kruys V, Gueydan C. The cold-inducible RNA-binding protein migrates from the nucleus to cytoplasmic stress granules by a methylation-dependent mechanism and acts as a translational repressor. Experimental cell research. 2007; 313: 4130-44.

24. Al-Fageeh MB, Smales CM. Cold-inducible RNA binding protein (CIRP) expression is modulated by alternative mRNAs. Rna. 2009; 15: 1164-76.

25. Wu L, Sun HL, Gao Y, Hui KL, Xu MM, Zhong H, et al. Therapeutic Hypothermia Enhances Cold-Inducible RNA-Binding Protein Expression and Inhibits Mitochondrial Apoptosis in a Rat Model of Cardiac Arrest. Molecular neurobiology. 2016.

26. Zhang HT, Xue JH, Zhang ZW, Kong HB, Liu AJ, Li SC, et al. Cold-inducible RNA-binding protein inhibits neuron apoptosis through the suppression of mitochondrial apoptosis. Brain research. 2015; 1622: 474-83.

27. Xue JH, Nonoguchi K, Fukumoto M, Sato T, Nishiyama H, Higashitsuji H, et al. Effects of ischemia and $\mathrm{H} 2 \mathrm{O} 2$ on the cold stress protein CIRP expression in rat neuronal cells. Free radical biology \& medicine. 1999; 27: 1238-44

28. Wellmann S, Buhrer C, Moderegger E, Zelmer A, Kirschner R, Koehne P, et al. Oxygen-regulated expression of the RNA-binding proteins RBM3 and CIRP by a HIF-1-independent mechanism. Journal of cell science. 2004; 117: 1785-94.

29. Nishiyama H, Xue JH, Sato T, Fukuyama H, Mizuno N, Houtani T, et al. Diurnal change of the cold-inducible RNA-binding protein (Cirp) expression in mouse brain. Biochemical and biophysical research communications. 1998; 245: 534-8.

30. Chang ET, Parekh PR, Yang Q, Nguyen DM, Carrier F. Heterogenous ribonucleoprotein A18 (hnRNP A18) promotes tumor growth by increasing protein translation of selected transcripts in cancer cells. Oncotarget. 2016; 7: 10578-93.

31. Negrini M, Nicoloso MS, Calin GA. MicroRNAs and cancer--new paradigms in molecular oncology. Current opinion in cell biology. 2009; 21: 470-9.

32. Camps C, Buffa FM, Colella S, Moore J, Sotiriou C, Sheldon H, et al. hsa-miR-210 Is induced by hypoxia and is an independent prognostic factor in breast cancer. Clinical cancer research : an official journal of the American Association for Cancer Research. 2008; 14: 1340-8.

33. Kulshreshtha R, Ferracin M, Wojcik SE, Garzon R, Alder H, Agosto-Perez FJ, et al. A microRNA signature of hypoxia. Molecular and cellular biology. 2007; 27: 1859-67.

34. Nallamshetty S, Chan SY, Loscalzo J. Hypoxia: a master regulator of microRNA biogenesis and activity. Free radical biology \& medicine. 2013; 64: 20-30.

35. Kou Y, Zheng WT, Zhang YR. Inhibition of miR-23 protects myocardial function from ischemia-reperfusion injury through restoration of glutamine metabolism. European review for medical and pharmacological sciences. 2016; 20: 4286-93.

36. Chang CY, Lui TN, Lin JW, Lin YL, Hsing CH, Wang JJ, et al. Roles of microRNA-1 in hypoxia-induced apoptotic insults to neuronal cells. Archives of toxicology. 2016; 90: 191-202.

37. Chen $\mathrm{Q}, \mathrm{Xu} \mathrm{J}, \mathrm{Li} \mathrm{L}, \mathrm{Li} \mathrm{H}$, Mao S, Zhang F, et al. MicroRNA-23a/b and microRNA-27a/b suppress Apaf-1 protein and alleviate hypoxia-induced neuronal apoptosis. Cell death \& disease. 2014; 5: e1132.

38. Whiteside G, Munglani R. TUNEL, Hoechst and immunohistochemistry triple-labelling: an improved method for detection of apoptosis in tissue sections--an update. Brain research Brain research protocols. 1998; 3: 52-3.

39. Rao JK, Letada P, Haverstick DM, Herman MM, Savory J. Modifications to the in situ TUNEL method for detection of apoptosis in paraffin-embedded tissue sections. Annals of clinical and laboratory science. 1998; 28: 131-7.

40. Chen $\mathrm{R}, \mathrm{Xu}$ M, Nagati JS, Hogg RT, Das A, Gerard RD, et al. The acetate/ACSS2 switch regulates HIF-2 stress signaling in the tumor cell microenvironment. PloS one. 2015; 10: e0116515.
41. Zhang $Q$, Wang $Y Z$, Zhang $W$, Chen $X$, Wang J, Chen J, et al. Involvement of Cold Inducible RNA-Binding Protein in Severe Hypoxia-Induced Growth Arrest of Neural Stem Cells In Vitro. Molecular neurobiology. 2016.

42. Tomimatsu T, Pena JP, Longo LD. Fetal hypercapnia in high-altitude acclimatized sheep: cerebral blood flow and cerebral oxygenation. Reproductive sciences. 2007; 14: 51-8.

43. Bickler PE, Julian D. Regional cerebral blood flow and tissue oxygenation during hypocarbia in geese. The American journal of physiology. 1992; 263: R221-5.

44. Huff J, Olmstead C, Stone JE. The effects of certain central nervous system stimulants upon the spontaneous activity of mice exposed to high altitude and low partial pressure of oxygen. The Journal of the Arkansas Medical Society. 1967; 64: 208-10.

45. Pena JP, Tomimatsu T, Hatran DP, McGill LL, Longo LD. Cerebral blood flow and oxygenation in ovine fetus: responses to superimposed hypoxia at both low and high altitude. The Journal of physiology. 2007; 578: 359-70.

46. Wright AD, Beazley MF, Bradwell AR, Chesner IM, Clayton RN, Forster PJ, et al. Medroxyprogesterone at high altitude. The effects on blood gases, cerebral regional oxygenation, and acute mountain sickness. Wilderness \& environmental medicine. 2004; 15: 25-31.

47. Lee HN, Ahn SM, Jang HH. Cold-inducible RNA-binding protein, CIRP, inhibits DNA damage-induced apoptosis by regulating p53. Biochemical and biophysical research communications. 2015; 464: 916-21.

48. Saito $\mathrm{K}$, Fukuda $\mathrm{N}$, Matsumoto $\mathrm{T}$, Iribe $\mathrm{Y}$, Tsunemi $\mathrm{A}$, Kazama $\mathrm{T}$, et al. Moderate low temperature preserves the stemness of neural stem cells and suppresses apoptosis of the cells via activation of the cold-inducible RNA binding protein. Brain research. 2010; 1358: 20-9.

49. Sakurai T, Itoh K, Higashitsuji H, Nonoguchi K, Liu Y, Watanabe H, et al. Cirp protects against tumor necrosis factor-alpha-induced apoptosis via activation of extracellular signal-regulated kinase. Biochimica et biophysica acta. 2006; 1763: 290-5.

50. Qiang X, Yang WL, Wu R, Zhou M, Jacob A, Dong W, et al. Cold-inducible RNA-binding protein (CIRP) triggers inflammatory responses in hemorrhagic shock and sepsis. Nature medicine. 2013; 19: 1489-95.

51. Sakurai $T$, Kashida $H$, Watanabe $T$, Hagiwara S, Mizushima $T$, Iijima $H$, et al. Stress response protein cirp links inflammation and tumorigenesis in colitis-associated cancer. Cancer research. 2014; 74: 6119-28.

52. Vordermark D. In regard to DAI et al.: inhibition of hypoxia inducible factor 1alpha causes oxygen-independent cytotoxicity and induces p53 independent apoptosis in glioblastoma cells. IJROBP 2003;55:1027-1036). International journal of radiation oncology, biology, physics. 2003; 57: 1196.

53. Luo W, Wang Y. HIF repressors under chronic hypoxia. Aging. 2016; 8: 418-9.

54. Doe MR, Ascano JM, Kaur M, Cole MD. Myc posttranscriptionally induces HIF1 protein and target gene expression in normal and cancer cells. Cancer research. 2012; 72: 949-57.

55. Iida Y, Aoki K, Asakura T, Ueda K, Yanaihara N, Takakura S, et al. Hypoxia promotes glycogen synthesis and accumulation in human ovarian clear cell carcinoma. International journal of oncology. 2012; 40: 2122-30.

56. Kappler M, Pabst U, Rot S, Taubert H, Wichmann H, Schubert J, et al. Normoxic accumulation of HIF1alpha is associated with glutaminolysis. Clinical oral investigations. 2017; 21: 211-24.

57. Kubis HP, Hanke N, Scheibe RJ, Gros G. Accumulation and nuclear import of HIF1 alpha during high and low oxygen concentration in skeletal muscle cells in primary culture. Biochimica et biophysica acta. 2005; 1745: 187-95.

58. Sudhagar S, Sathya S, Lakshmi BS. Rapid non-genomic signalling by 17beta-oestradiol through c-Src involves mTOR-dependent expression of HIF-1alpha in breast cancer cells. British journal of cancer. 2011; 105: 953-60.

59. Lichner Z, Mejia-Guerrero S, Ignacak M, Krizova A, Bao TT, Girgis AH, et al. Pleiotropic action of renal cell carcinoma-dysregulated miRNAs on hypoxia-related signaling pathways. The American journal of pathology. 2012; 180: $1675-87$

60. Sun $\mathrm{Y}$, Tao $\mathrm{C}$, Huang $\mathrm{X}$, He $\mathrm{H}$, Shi $\mathrm{H}$, Zhang $\mathrm{O}$, et al. Metformin induces apoptosis of human hepatocellular carcinoma HepG2 cells by activating an AMPK/p53/miR-23a/FOXA1 pathway. OncoTargets and therapy. 2016; 9: 2845-53.

61. Peng F, Zhang H, Du Y, Tan P. miR-23a promotes cisplatin chemoresistance and protects against cisplatin-induced apoptosis in tongue squamous cell carcinoma cells through Twist. Oncology reports. 2015; 33: 942-50.

62. Lee YE, Hong CY, Lin YL, Chen RM. MicroRNA-1 participates in nitric oxide-induced apoptotic insults to MC3T3-E1 cells by targeting heat-shock protein-70. International journal of biological sciences. 2015; 11: 246-55. 